NASA/CR-2002-211662

ICASE Report No. 2002-22

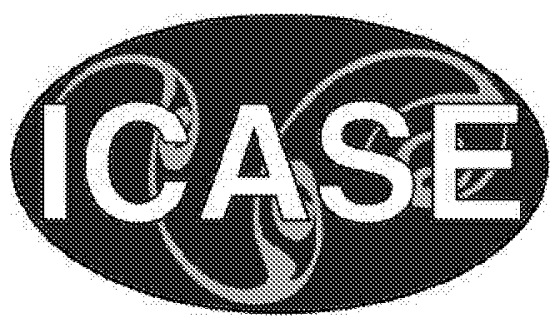

\title{
Force Evaluation in the Lattice Boltzmann Method Involving Curved Geometry
}

Renwei Mei, Dazhi Yu, and Wei Shyy

University of Florida, Gainesville, Florida

Li-Shi Luo

ICASE, Hampton, Virginia 


\section{The NASA STI Program Office ... in Profile}

Since its founding, NASA has been dedicated to the advancement of aeronautics and space science. The NASA Scientific and Technical Information (STI) Program Office plays a key part in helping NASA maintain this important role.

The NASA STI Program Office is operated by Langley Research Center, the lead center for NASA's scientific and technical information. The NASA STI Program Office provides access to the NASA STI Database, the largest collection of aeronautical and space science STI in the world. The Program Office is also NASA's institutional mechanism for disseminating the results of its research and development activities. These results are published by NASA in the NASA STI Report Series, which includes the following report types:

- TECHNICAL PUBLICATION. Reports of completed research or a major significant phase of research that present the results of NASA programs and include extensive data or theoretical analysis. Includes compilations of significant scientific and technical data and information deemed to be of continuing reference value. NASA's counterpart of peer-reviewed formal professional papers, but having less stringent limitations on manuscript length and extent of graphic presentations.

- TECHNICAL MEMORANDUM. Scientific and technical findings that are preliminary or of specialized interest, e.g., quick release reports, working papers, and bibliographies that contain minimal annotation. Does not contain extensive analysis.

- CONTRACTOR REPORT. Scientific and technical findings by NASA-sponsored contractors and grantees.
- CONFERENCE PUBLICATIONS. Collected papers from scientific and technical conferences, symposia, seminars, or other meetings sponsored or cosponsored by NASA.

- SPECIAL PUBliCATION. Scientific, technical, or historical information from NASA programs, projects, and missions, often concerned with subjects having substantial public interest.

- TECHNICAL TRANSLATION. Englishlanguage translations of foreign scientific and technical material pertinent to NASA's mission.

Specialized services that complement the STI Program Office's diverse offerings include creating custom thesauri, building customized data bases, organizing and publishing research results ... even providing videos.

For more information about the NASA STI Program Office, see the following:

- Access the NASA STI Program Home Page at http://www.sti.nasa.gov

- Email your question via the Internet to help@sti.nasa.gov

- Fax your question to the NASA STI Help Desk at (301) 621-0134

- Telephone the NASA STI Help Desk at (301) 621-0390

- Write to: NASA STI Help Desk NASA Center for AeroSpace Information 7121 Standard Drive Hanover, MD 21076-1320 
NASA/CR-2002-211662

ICASE Report No. 2002-22

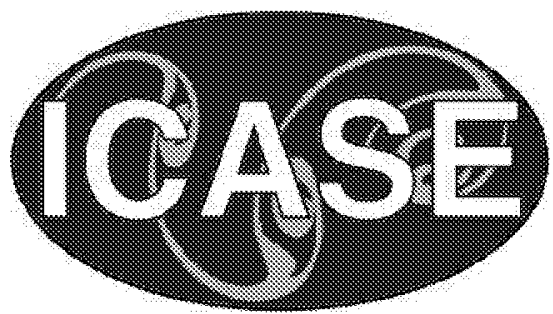

\section{Force Evaluation in the Lattice Boltzmann Method Involving Curved Geometry}

Renwei Mei, Dazhi Yu, and Wei Shyy

University of Florida, Gainesville, Florida

Li-Shi Luo

ICASE, Hampton, Virginia

ICASE

NASA Langley Research Center

Hampton, Virginia

Operated by Universities Space Research Association 
Available from the following:

NASA Center for AeroSpace Information (CASI)

7121 Standard Drive

Hanover, MD 21076-1320

National Technical Information Service (NTIS)

5285 Port Royal Road

Springfield, VA 22161-2171

(301) 621-0390

(703) $487-4650$ 


\title{
FORCE EVALUATION IN THE LATTICE BOLTZMANN METHOD INVOLVING CURVED GEOMETRY
}

\author{
RENWEI MEI*, DAZHI YU', WEI SHYY ${ }^{\ddagger}$, AND LI-SHT LUO
}

\begin{abstract}
The present work investigates two approaches for force evaluation in the lattice Boltzmann equation: the momentum-exchange method and the stress-integration method on the surface of a body. The boundary condition for the particle distribution functions on curved geometries is handled with second order accuracy based on our recent works. The stress-integration method is computationally laborious for two-dimensional flows and in general difficult to implement for three-dimensional flows, while the momentumexchange method is reliable, accurate, and easy to implement for both two-dimensional and three-dimensional flows. Several test cases are selected to evaluate the present methods, including: (i) two-dimensional pressuredriven channel flow; (ii) two-dimensional uniform flow past a column of cylinders; (iii) two-dimensional flow past a cylinder asymmetrically placed in a channel (with vortex shedding); (iv) three-dimensional pressuredriven flow in a circular pipe; and (v) three-dimensional flow past a sphere. The drag evaluated by using the momentum-exchange method agrees well with the exact or other published results.
\end{abstract}

Key words. lattice Boltzmann method, force evaluation on fluid-solid interface, momentum-exchange method, stress-integration method, boundary condition for curved geometries, accuracy, 3-D flows

Subject classification. Fluid Mechanics

\section{Introduction.}

1.1. Background of the lattice Boltzmann equation method. The method of lattice Boltzmann equation (LBE) solves the microscopic kinetic equation for particle distribution function $f(x, \xi, t)$, where $\xi$ is the particle velocity, in phase space $(\boldsymbol{x}, \boldsymbol{\xi})$ and time $t$, from which the macroscopic quantities (flow mass density $\rho$ and velocity $\boldsymbol{u}$ ) are obtained through moment integration of $f(x, \xi, t)$. Because the solution procedure is explicit, easy to implement and parallelize, the LBE method has increasingly become an attractive alternative computational method for solving fluid dynamics problems in various systems [1, 2, 3, 4]. The most widely used lattice Boltzmann equation $[1,2,3,4]$ is a discretized version of the model Boltzmann equation with a single relaxation time approximation due to Bhatnagar, Cross, and Krook (BGK model) $[5]$,

$$
\partial_{t} f+\xi \cdot \nabla f=\frac{1}{\lambda}\left[f-f^{(0)}\right]
$$

where $f^{(0)}$ is the Maxwell-Boltzmann equilibrium distribution function and $\lambda$ is the relaxation time. The mass density $\rho$ and momentum density $\rho u$ are the first $(D+1)$ hydrodynamic moments of the distribution

"Department of Aerospace Engineering, Mechanics \& Engineering Science, University of Florida, Gainesville, FL $32611-6250$ (email address: rwm@aero.ufl.edu).

Department of Aerospace Engineering, Mechanics \& Engineering Science, University of Florida, Gainesville, FL $32611-6250$ (email address: ydzQaero.ufl.edu).

$\$$ Department of Aerospace Engineering, Mechanics \& Engineering Science, University of Florida, Gainesville, FL 32611-6250 (email address: ws@aero.ufledu).

ICASE, Mail Stop 132C, NASA Langley Research Center, 3 West Reid Street, Building 1152, Hampton, VA 23681-2199 (email address: luodicase.edu). This research was supported by the National Aeronantics and Space Administration under NASA Contract No. NAS1-97046 while the author was in residence at ICASE, NASA Langley Research Center, Hampton, VA $23681-2199$. 
function $f$ and $f^{(0)}$ in $D$ dimensions. It can be shown that the particle velocity space $\xi$ can be discretized and reduced to a very small set of discrete velocities $\left\{\xi_{\alpha} \mid \alpha=1,2, \ldots, b\right\}$, and the hydrodynamic moments of $f$ and $f^{(0)}$ as well as their fluxes can be preserved exactly, because the moment integral can be replaced by quadrature exactly up to a certain order in $\xi[6,7,8,9]$. With velocity space $\xi$ properly discretized, Eq. (1.1) reduces to a discrete velocity model of the Boltzmann equation

$$
\partial_{t} f_{\alpha}+\xi_{\alpha} \cdot \nabla f_{\alpha}=\frac{1}{\lambda}\left[f_{\alpha}-f_{\alpha}^{(0)}\right]
$$

In the above equation, $f_{\alpha}(x, t) \equiv f\left(x, \xi_{\alpha}, t\right)$ and $f_{\alpha}^{(0)}(x, t) \equiv f^{(0)}\left(x, \xi_{\alpha}, t\right)$ are the distribution function and the equilibrium distribution function of the $\alpha$ th discrete velocity $\xi_{\alpha}$, respectively. Equation (1.2) is then discretized in space $x$ and time $t$ into

$$
f_{\alpha}\left(x_{i}+e_{\alpha} \delta_{i}, t+\delta_{t}\right)-f_{\alpha}\left(x_{i}, t\right)=-\frac{1}{\tau}\left[f_{\alpha}\left(x_{i}, t\right)-f_{\alpha}^{(\mathrm{eq})}\left(x_{i}, t\right)\right]
$$

where $\tau=\lambda / \delta_{t}$ is the dimensionless relaxation time and $e_{\alpha}$ is a discrete velocity vector. The coherent discretization of space and time is done in such a way that $\delta x=e_{\alpha} \delta_{t}$ is always the displacement vector from a lattice site to one of its neighboring sites. The equilibrium distribution function $f_{\alpha}^{(\mathrm{e})}\left(x_{i}, t\right)$ in the lattice Boltzmann equation (1.3) is obtained by expanding the Maxwell-Boltzmann distribution function in Taylor series of $\boldsymbol{u}$ up to second order $[6,7]$, and can be expressed in general as

$$
f_{\alpha}^{(\mathrm{eq})}=w_{\alpha} \rho\left[1+\frac{3}{c^{2}}\left(e_{\alpha} \cdot u\right)+\frac{9}{2 c^{4}}\left(e_{\alpha} \cdot u\right)^{2}-\frac{3}{2 c^{2}} u^{2}\right],
$$

where $c \equiv \delta_{x} / \delta_{t} ; \delta_{x}$ is the lattice constant of the underlying lattice space; and coefficient $w_{\alpha}$ depends on the discrete velocity set $\left\{e_{\alpha}\right\}$ in $D$ spatial dimensions. In what follows, we shall use the lattice units of $\delta_{x}=1$ and $\delta_{t}=1$. The Appendix provides the details of coefficient $w_{\alpha}$ and the discrete velocity set $\left\{e_{\alpha}\right\}$ for the twodimensional nine-velocity model (D2Q9) and the three-dimensional nineteen-velocity model (D3Q19) [10]. Figure 1 shows the discrete velocity sets of the two models. It sbould be pointed out that there exist other discrete velocity sets $\left\{e_{\alpha}\right\}$ that have the sufficient symmetry for hydrodynamics [6, 7$]$. A comparative study of three three-dimensional LBE models including the fifteen-velocity model (D3Q15), the nineteen-velocity model (D3Q19), and the twenty-seven-velocity model (D3Q27), in terms of accuracy and computational efficiency has been conducted by Mei et al. [11]. It was found that the nineteen-velocity model (D3019) offers a better combination of computational stability and accuracy. The D2Q9 and D3Q19 models will be used in this study for force evaluation in two-dimensional and three-dimensional flows, respectively. Equation (1.3) is conveniently solved in two steps

$$
\begin{aligned}
\text { collision: } & f_{\alpha}\left(x_{i}, t\right)=f_{\alpha}\left(x_{i}, t\right)-\frac{1}{\tau}\left[f_{\alpha}\left(x_{i}, t\right)-f_{\alpha}^{(\text {eq })}\left(x_{i}, t\right)\right], \\
\text { streaming: } & f_{\alpha}\left(x_{i}+e_{\alpha} \delta_{t}, t+\delta_{t}\right)=\tilde{f}_{\alpha}\left(x_{i}, t\right),
\end{aligned}
$$

which is known as the LBGK scheme $[1,2]$. The collision step is completely local and the streaming step is uniform and requires little computational effort, which makes Eq. (1.5) ideal for parallel implementation. The simplicity and compact nature of the LBGK scheme, however, necessitate the use of the square lattices of constant spacing $\left(\delta_{x}=\delta_{y}\right)$, and consequently lead to the unity of the local Courant-Friedrichs-Lewy number, because $\delta_{t}=\delta_{x}=1$.

1.2. Boundary condition for a curved geometry in the LBE method. Consider a part of an arbitrary curved wall geometry, as shown in Fig. 2 , where the filled small circles on the boundary, $\boldsymbol{x}_{w}$, denote 

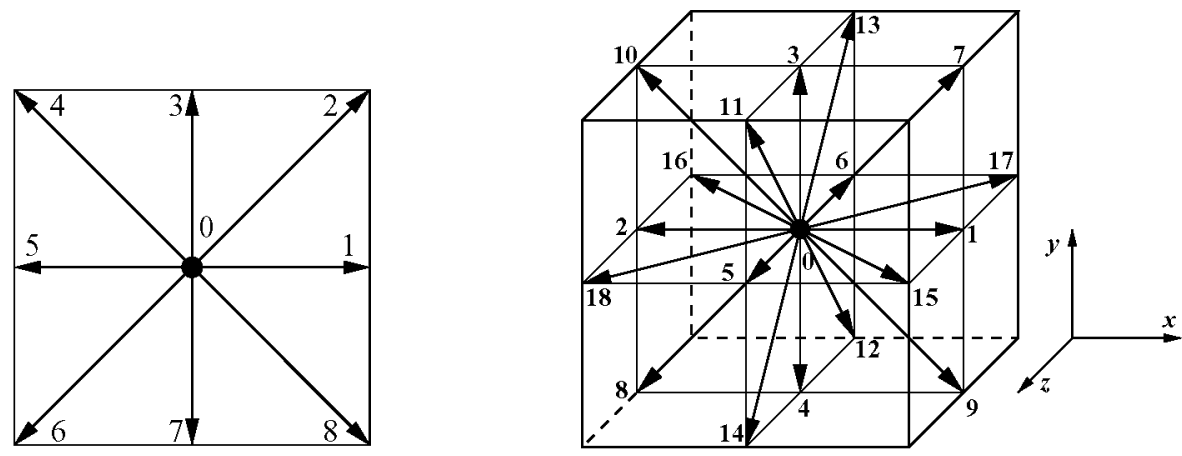

FIG. 1. Discrete velocity set $\left\{e_{\alpha}\right\}$. (left) Two-dimensional nine-velocity (D2Q9) model. (right) Three-dimensional nineteen-velocity (D3Q19) model.

the intersections of the boundary with various lattice-to-lattice links. The fraction of an intersected link in the fluid region, $\Delta$, is defined by

$$
\Delta=\frac{\left\|x_{f}-x_{w}\right\|}{\left\|x_{f}-x_{b}\right\|} .
$$

Obviously the horizontal or vertical distance between $x_{b}$ and $x_{w}$ is $\Delta \delta_{x}$ on the square lattice, and $0 \leq \Delta \leq 1$. In Eq. (1.5b), the value of $\tilde{f}_{\alpha}\left(x_{i}, t\right)$ needs to be constructed according to the location of the boundary and the boundary conditions, if the grid point $x_{i}=x_{b}$ lies beyond the boundary. In the past, the bounceback boundary condition has been use to deal with a solid boundary in order to approximate the no-slip boundary condition at the solid boundary $[12,13,14,15,16,17,18,19,20,21,22,23,24]$. However, it is well understood that this bounce-back boundary condition satisfies the no-slip boundary condition with a second-order accuracy (for the Couette and Poiseuille flows) at the location one half lattice spacing $(\Delta=1 / 2)$ outside of a boundary node where the bounce-back collision takes place; and this is only true with simple boundaries of straight lines parallel to the lattice grid $[19,20,21]$. For a curved geometry, simply placing the boundary halfway between two nodes will alter the geometry on the grid level and degrade the accuracy of the flow field and the force on the body at finite and higher Reynolds number. To circumvent this difficulty, Mei and Shyy solved Eq. (1.2) in curvilinear coordinates using a finite difference method to compute $f_{\alpha}$ [25]. He and Doolen used body-fitted curvilinear coordinates with interpolation throughout the entire mesh, except at the boundaries where the bounce-back boundary condition is used [26]. In the recent works of Filippova and Hänel [27] and Mei et al. [28, 11], a second-order accurate boundary condition for curved geometry was developed in conjunction with the use of Cartesian grids in order to retain the advantages of the LBE method. An interpolation scheme is employed only at the boundaries to obtain $\tilde{f}_{\alpha}\left(\boldsymbol{x}_{i}, t\right)$. The detailed assessment on the impact of the boundary condition on the accuracy of the flow field has been given in Ref. [28] for some two-dimensional flows and in Ref. [11] for some three-dimensional flows.

Because the bounce-back type boundary conditions play an important role in lattice Boltzmann simulations, it is important for us to understand how the boundary conditions work. First of all, one must realize that it is impossible for any kinetic numerical scheme to impose a given velocity (the Dirichlet boundary condition) on a given grid node, because the Knudsen layer type of phenomena [29, 30, 31] would be manifested in kinetic schemes $[32,19,20,21]$. For example, in the Poiseuille and the Couette flows, the location where hydrodynamic boundary conditions are satisfied are one-half grid spacing away from the boundary grids where the bounce-back boundary conditions are imposed $[19,20,21]$. For flows around an arbitrary shaped body analytical solutions do not exist. Nevertheless, substantial evidence shows that the bounce- 


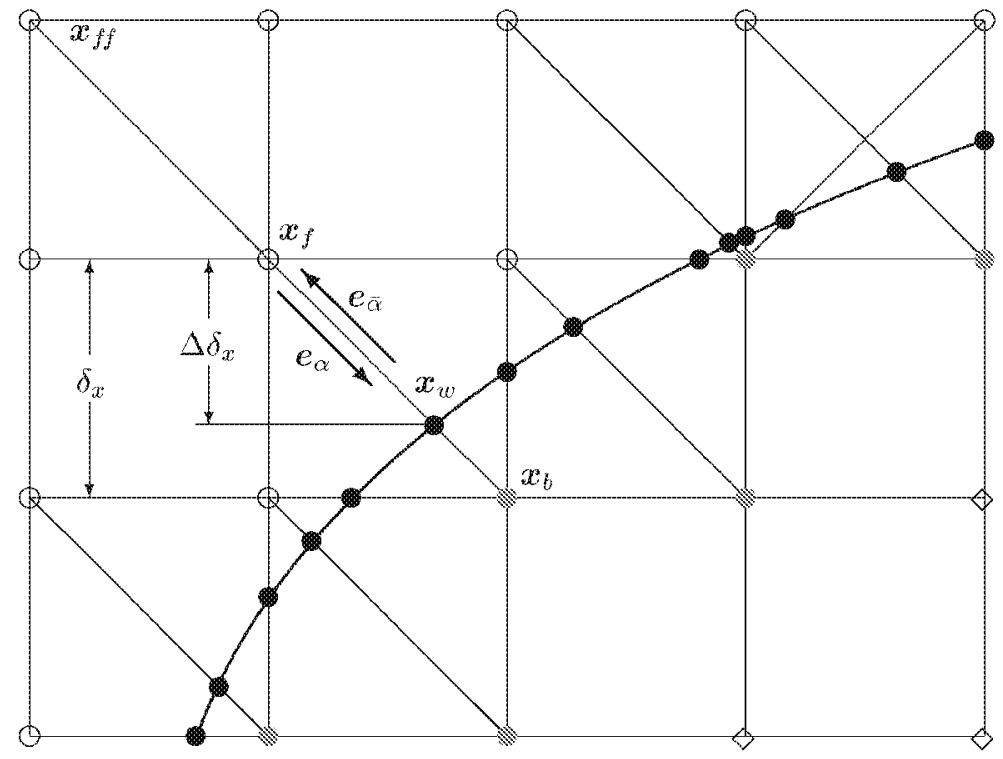

FIG. 2. Layout of the regularly spaced lattices and curved wall boundary. The circles (o), dises ( $)$, shaded discs (s), and diamonds $(\diamond)$ denote fluid nodes, boundary locations $\left(\boldsymbol{x}_{w}\right)$, solid nodes which are also boundary nodes $\left(x_{b}\right)$ inside solid, and solid nodes, respectively.

back boundary conditions combined with interpolations, and including the one-half grid spacing correction at boundaries, are in fact second-order accurate and capable of handling curved boundaries [26, 23, 24, 33]. This point is also demonstrated in the present work.

1.3. Force evaluation and related works. In spite of numerous improvement for the LBE method during the last several years, one important issue that has not been systematically studied is the accurate determination of the fluid dynamic force involving curved boundaries. Needless to say, accurate evaluation of the force is crucial to the study of fluid dynamics, especially in fluid-structure interaction. Several force evaluation schemes, including momentum exchange $[14,16]$ and integration of surface stress $[26,34]$, have been used to evaluate the fluid dynamic force on a curved body in the context of the LBE method.

He and Doolen [26] evaluated the force by integrating the total stress on the surface of the cylinder and the components of the stress tensor were obtained by taking respective velocity gradients. Even though a body-fitted grid was used, an extrapolation was needed to obtain the stress in order to correct the halfgrid-cell spacing effect due to the bounce-back boundary condition. Filippova and Hänel [27] developed a second-order accurate boundary condition for curved boundaries. However, the fluid dynamics force on a circular cylinder asymmetrically placed in a two-dimensional chamel was obtained by integrating the pressure and deviatoric stresses on the surface of the cylinder by extrapolating from the nearby Cartesian grids to the solid boundary [27,34]. To gain insight into the method of surface stress integration, it is instructive to examine the variation of the pressure on the surface of a circular cylinder at finite Reynolds number obtained by using the LBE method for flow over a column of cylinders (see Ref. [28], and Sec. 3.2). Figure 3 shows the pressure coefficient

$$
C_{P}=\frac{p-p_{\infty}}{\frac{1}{2} \rho U^{2}}
$$




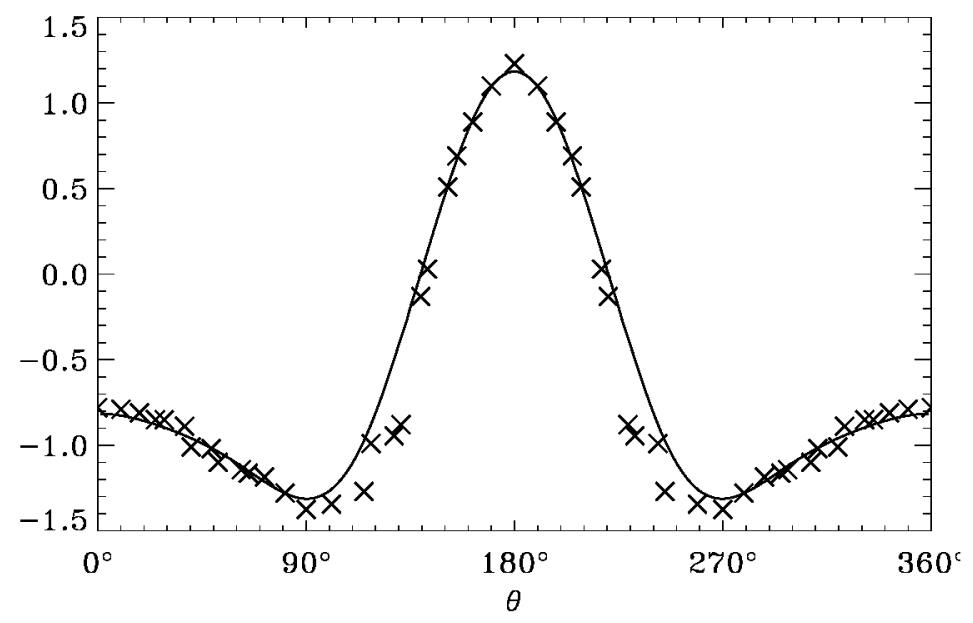

FIG. 3. Flow past a column of $2 D$ circular cylinders. Distribution of the pressure coefficient $C p$ on the surface of a $2 D$ circular cylinder of radius $r=6.6$, and center-to-center distance $H / r=20$. The stagnation point is located at $\theta=180^{\circ}$. The LBE result denoted by symbols $x$ is obtained with $\tau=0.6$ and $\mathrm{Re}=40$. The solid line is the result obtained by using a $3 D$ multi-block, body-fitted grid, and pressure-based Nonier-Stokes solver with a much finer resolution.

on the surface obtained by using second-order extrapolation, where $p_{\infty}$ is the far upstream pressure. Only those boundary points, $x_{w}$, intersected by the horizontal or vertical velocities, i.e., $e_{1}, e_{3}, e_{5}$, and $e_{7}$, are considered in the result given by Fig. 3. If the boundary points intersected by the links in the diagonal velocities, i.e., $e_{2}, e_{4}, e_{6}$, and $e_{8}$, are also considered, the variation of $C_{P}$ would be more noisy. The components of the deviatoric stress tensor show a similar noisy pattern. It is not clear how the noise in the pressure and stresses affect the accuracy of the fluid dynamic force in the stress-integration method. While the programming in the extrapolation and integration is manageable in two-dimensional (3D) cases, it is rather laborious in three-dimensional cases. In Fig. 3, the LBE result of $C_{P}(\theta)$ (indicated by symbol $x$ ) is compared with that obtained by using a 3D multiblock, body-fitted coordinates, and pressure-based Navier-Stokes solver $[35,36,37]$ with a much finer resolution: 201 points around the cylinder and the smallest grid size along the radial direction $d r=0.026$ (relative to $r=1$ ). Not surprisingly, the result obtained by using the Navier-Stokes solver with body-fitted grid and high resolution is smoother than the LBE result with a Cartesian grid of coarser resolution. Nevertheless, the LBE solution still essentially agrees with the Navier-Stokes solution.

Instead of the stress-integration method, Ladd used the momentum-exchange method to compute the fluid force on a sphere in suspension flow [14]. In the flow simulation using the bounce-back boundary condition, the body is effectively replaced by a series of stairs. Each segment on the surface has an area of unity for a cubic lattice. The force on each link [halfway between two lattices at $x_{f}$ and $x_{b}=\left(x_{f}+e_{\alpha} \delta_{t}\right)$ in which $x_{b}$ resides in the solid region] results from the momentum exchange (per unit time) between two opposing directions of the neighboring lattices

$$
\frac{1}{\delta_{t}}\left[e_{\alpha} f_{\alpha}\left(x_{f}\right)-e_{\bar{\alpha}} f_{\bar{\alpha}}\left(x_{f}+e_{\alpha} \delta_{t}\right)\right]
$$

in which $e_{\bar{\alpha}} \equiv-e_{\alpha}$. Whereas the momentum-exchange method is very easy to implement computationally, its applicability and accuracy for a curved boundary have not been systematically studied. To recapitulate, 
there are two major problems associated with the method of surface stress integration. First, the components of stress tensor are often noisy on a curved surface due to limited resolution near the body and the use of Cartesian grids. The accuracy of such a method has not been addressed in the literature. Second, the implementation of the extrapolation for Cartesian components of the stress tensor to the boundary surface and the integration of the stresses on the surface of a three-dimensional geometry are very laborious in comparison with the intrinsic simplicity of the lattice Boltzmann simulations for flow field. The problems associated with the method of momentum exchange are as follows. (a) The scheme was proposed for the case with $\Delta=1 / 2$ at every boundary intersection $\boldsymbol{x}_{w}$. Whether this scheme can be applied to the cases where $\Delta \neq 1 / 2$, when, for example, the boundary is not straight, needs to be investigated. (b) As in the case of stress-integration method, the resolution near a solid body is often limited and the near wall flow variables can be noisy. If one uses the momentum-exchange method to compute the total force, it is not clear what the adequate resolution is to obtain reliable fluid dynamic force on a bluff body at a given (moderate) value of Reynolds number, say, Re $\approx O\left(10^{2}\right)$.

1.4. Scope of the present work. In what follows, two methods for the force evaluation, i.e., the stress-integration and the momentum-exchange methods, will be described in detail. The shear and normal stresses on the wall in a pressure driven channel flow will be first examined to assess the suitability of the momentum-exchange method when $\Delta \neq 1 / 2$ and analyze the errors incurred. The results on the drag force for flow over a column of circular cylinders using these two methods will be subsequently assessed for the consistency. The drag coefficient at $R e=100$ are compared with the result of Fornberg [38] obtained by using a second-order accurate finite difference scheme with sufficient grid resolution. For flow over a cylinder asymmetrically placed in a channel at $\mathrm{Re}=100$, the unsteady drag and lift coefficients are computed and compared with the results in the literature. The momentum-exchange method is further evaluated for threedimensional fully developed pipe flow and for a uniform flow over a two-dimensional array of spheres at finite Reynolds number. We found that the simple momentum-exchange method for force evaluation gives fairly reliable results for the two-dimensional and three-dimensional flows.

\section{Methods for Force Evaluation in LBE Method.}

2.1. Second-order accurate no-slip boundary condition for curved geometry. The analysis of boundary conditions for a curved boundary in the lattice Boltzmann equation is accomplished by applying the Chapman-Enskog expansion for the distribution function at the boundary. The following approximation for the post-collision distribution function on the right-hand side of $\mathrm{Eq}$. (1.5b) can lead to a second-order accurate no-slip boundary condition $[11,27,28]$

$$
\tilde{f}_{\bar{\alpha}}\left(x_{b}, t\right)=(1-\chi) \tilde{f}_{\alpha}\left(x_{f}, t\right)+\chi f_{\alpha}^{*}\left(x_{b}, t\right)+2 w_{\alpha} \rho \frac{3}{c^{2}} e_{\bar{\alpha}} \cdot u_{w},
$$

where

$$
\begin{aligned}
f_{\alpha}^{*}\left(x_{b}, t\right) & =w_{\alpha} \rho\left(x_{f}, t\right)\left[1+\frac{3}{c^{2}}\left(e_{\alpha} \cdot u_{b f}\right)+\frac{9}{2 c^{4}}\left(\boldsymbol{c}_{\alpha} \cdot u_{f}\right)^{2}-\frac{3}{2 c^{2}} u_{f}^{2}\right] \\
& =f_{\alpha}^{(\mathrm{eq})}\left(x_{f}, t\right)+w_{\alpha} \rho\left(\boldsymbol{x}_{f}, t\right) \frac{3}{c^{2}} e_{\alpha} \cdot\left(u_{b f}-u_{f}\right)
\end{aligned}
$$

and

$$
\begin{aligned}
& u_{b f}=u_{f f}=u_{f}\left(x_{f}+e_{\bar{\alpha}} \delta_{t}, t\right), \quad \chi=\frac{(2 \Delta-1)}{(\tau-2)}, \quad 0 \leq \Delta<\frac{1}{2} \\
& u_{b f}=\frac{1}{2 \Delta}(2 \Delta-3) u_{f}+\frac{3}{2 \Delta} u_{w}, \quad \chi=\frac{(2 \Delta-1)}{(\tau+1 / 2)}, \quad \frac{1}{2} \leq \Delta<1 .
\end{aligned}
$$


The above treatment is applicable for both the two-dimensional and three-dimensional lattice Boltzmann models.

By substitution of Eq. (2.2), Eq. (2.1) becomes

$$
\begin{array}{r}
\tilde{f}_{\bar{\alpha}}\left(x_{b}, t\right)=\tilde{f}_{\alpha}\left(x_{f}, t\right)-\chi\left[\tilde{f}_{\alpha}\left(x_{f}, t\right)-f_{\alpha}^{(\mathrm{e})}\left(x_{f}, t\right)\right] \\
+w_{\alpha} \rho\left(x_{f}, t\right) \frac{3}{c^{2}} e_{\alpha} \cdot\left(u_{b f}-u_{j}-2 u_{w}\right) .
\end{array}
$$

Thus, the above treatment of curved boundaries can be thought as a modification of the relaxation (the viscous effect) near the wall (with the relaxation parameter $\chi$ ), in additional to a forcing term accounting for the momentum-exchange effect due to the wall.

2.2. Force evaluation based on stress integration. He and Doolen [26] evaluated the force by integrating the total stresses on the boundary of the cylinder $\partial \Omega$,

$$
F=\int_{\partial \Omega} d A \hat{n} \cdot\left\{-p l+\rho v\left[(\nabla: u)+(\nabla: u)^{\top}\right]\right\}
$$

where $\hat{n}$ is the unit out normal vector of the boundary $\partial \Omega, \|$ is the identity tensor of second rank, $\nabla: u$ denotes the second rank tensor whose components are $\partial_{i} w_{j}$, and $T$ is the tranpose operator. In Ref. [26], a body-fitted coordinate system together with grid stretching was used such that a large number of grids can be placed near the body to yield a reliable velocity gradient $\partial_{i} u_{j}$. In general, since $u$ is not the primary variable in the LBE simulations and the evaluation of $u$ using $\sum_{\alpha} e_{\alpha} f_{\alpha}$ based on $f_{\alpha}$ 's suffers the loss of accuracy due to the cancellation of two close numbers in $f_{\alpha}$ 's the evaluation of the derivative $\partial_{2} u_{j}$ will result in further degradation of the accuracy. Filippova [34] used a similar integration scheme to obtain the dynamic force on the body for the force on a circular cylinder [27] except that the deviatoric stresses were evaluated using the non-equilibrium part of the particle distribution function [see Eq. (2.7) below], However, since a Cartesian grid was used, the stress vectors on the surface of the body (with arbitrary $\Delta$ ) have to be computed through an extrapolation procedure based upon the information in the flow field. This leads to further loss of accuracy for a finite lattice size $\delta_{x}$ when the shear-layer near the wall is not sufficiently resolved.

In Eq. (2.5), the pressure $p$ can be easily evaluated using the equation of state $p=c_{s}^{2} \rho$. For D2Q9 and D3Q19 models, $c_{s}^{2}=1 / 3$ so that $p=\rho / 3$. The deviatoric stress for two-dimensional incompressible flow

$$
\tau_{i j}=\rho \nu\left(\partial_{i} u_{j}+\partial_{j} u_{i}\right)
$$

can be evaluated using the non-equilibrium part of the distribution function $f_{\alpha}^{(\text {(neq) }}=\left[f_{\alpha}-f_{\alpha}^{(\text {eq })}\right]$

$$
\tau_{i j}=\left(1-\frac{1}{2 \tau}\right) \sum_{\alpha} f_{\alpha}^{(\text {req) }}(x, t)\left(e_{\alpha, i} e_{\alpha, j}-\frac{1}{D} e_{\alpha} \cdot e_{\alpha} \delta_{i j}\right)
$$

where $e_{\alpha, i}$ and $\epsilon_{\alpha, j}$ are $i$ th and $j$ th Cartesian component of the discrete velocity $e_{\alpha}$, respectively. For the flow past a circular cylinder, a separate set of surface points on the cylinder can be introduced in order to carry out the numerical integration given by Eq. (2.5). The values of the pressure and each of the six components of the symmetric deviatoric stress tensor on the surface points can be obtained using a secondorder extrapolation scheme based on the values of $p$ and $\tau_{i j}$ at the neighboring fluid lattices. The force exerting on the boundary $2 \Omega$ is computed as

$$
F=\int_{\partial \Omega} d A \hat{n} \cdot\left\{-p \mid+\rho \nu\left[(\nabla: u)+(\nabla: u)^{\mathrm{T}}\right]\right\}_{\text {extrapolated }} .
$$

It is worth commenting here that for the two-dimensional flow past a cylinder, nearly half of the length of the entire code is taken up by the above force evaluation procedure. 
2.3. Method based on the momentum exchange. In order to employ the momentum-exchange method efficiently, two scalar arrays, $w(i, j)$ and $w_{b}(i, j)$ are introduced. A value of 0 is assigned to $w(i, j)$ for the lattice site $(i, j)$ that are occupied by fluid; a value of 1 is assigned to $w(i, j)$ for those lattice nodes inside the solid body. The array $w_{b}(i, j)$ is set to zero everywhere except for those boundary nodes, $x_{b}$, where a value of 1 is assigned. For a given nonzero velocity $e_{\alpha}, e_{\bar{\alpha}}$ denotes the velocity in the opposite direction, i.e., $e_{\bar{\alpha}} \equiv-e_{\alpha}$ (see Fig. 2). For a given boundary node $x_{b}$ inside the solid region with $w_{b}(i, j)=1$ and $w(i, j)=1$, the momentum exchange with all possible neighboring fluid nodes over a time step $\delta_{t}=1$ is

$$
\sum_{\alpha \neq 0} e_{\alpha}\left[\tilde{f}_{\alpha}\left(x_{b}, t\right)+\tilde{f}_{\bar{\alpha}}\left(x_{b}+e_{\bar{\alpha}} \delta_{t}, t\right)\right]\left[1-w\left(x_{b}+e_{\bar{\alpha}} \delta_{t}\right)\right]
$$

Simply summing the contribution over all boundary nodes $x_{b}$ belonging to the body, the total force (acted by the solid body on the fluid) is obtained as

$$
\boldsymbol{F}=\sum_{\text {all } \boldsymbol{x}_{b}} \sum_{\alpha \neq 0} \boldsymbol{e}_{\alpha}\left[\tilde{f}_{\alpha}\left(x_{b}, t\right)+\tilde{f}_{\bar{\alpha}}\left(x_{b}+\boldsymbol{e}_{\bar{\alpha}} \delta_{\bar{t}}, t\right)\right]\left[1-w\left(x_{b}+\boldsymbol{e}_{\bar{\alpha}} \delta_{t}\right)\right]
$$

In the momentum-exchange method the force $F$ is evaluated after the collision step is carried out and the value of $\tilde{f}_{\bar{\alpha}}$ at the boundary given by Eq. (2.1) has been evaluated. The momentum exchange occurs during the subsequent streaming step when $\tilde{f}_{\alpha}\left(x_{b}, t+\delta_{t}\right)$ and $\tilde{f}_{\alpha}\left(x_{f}, t+\delta_{t}\right)$ move to $x_{f}$ and $x_{b}$, respectively. As mentioned in the introductory section, the effect of the variable $\Delta$ is not explicitly included, but it is implicitly taken into account in the determination of $\tilde{f}_{a}\left(\boldsymbol{x}_{b}, t+\delta_{t}\right)$. The applicability of Eq. (2.9) will be examined and validated.

Clearly, the force is proportional to the number of boundary nodes $x_{b}$ in the above formula of $F$ and the number of the boundary nodes increase linearly with the size of the body in a two-dimensional flow. However, since the force is normalized by $\rho U^{2} r$ in the formula for $C_{D}$ in two-dimensions [see Eq. (3.9)], the drag coefficient $C_{D}$ should be independent of $r$.

3. Results and Discussions. For straight walls, there is no doubt that Eq. (2.5) together with the equation of state for pressure and Eq. (2.7) for $\tau_{i j}$ gives accurate results for the force provided that the $f_{\alpha}$ 's are accurately computed. To demonstrate the correctness of Eq. (2.9) based on the momentum exchange for an arbitrary $\Delta$, we first consider the pressure driven channel flow (see Fig. 4) for which exact solutions for the velocity and stresses are known. The second case considered is the two-dimensional flow past a column of circular cylinders at Reynolds number $\mathrm{Re}=100$ and $H / r=20$, where $H$ is the distance between the centers of two adjacent cylinders. The values of the drag computed using the two force evaluation methods are then compared with the result of Fornberg [38]. The dependence of the drag on the radius $r$ in the momentumexchange method is examined to assess the reliability of this method. The third case is the two-dimensional flow over a circular cylinder that is asymmetrically placed in a channel at $R e=100$ (with vortex shedding). The time dependence of the drag and lift coefficients is compared with results in the literature.

We also consider two cases of three-dimensional flow. The first case is the pressure driven flow in a circular pipe for which the exact solutions for both the velocity profile and the wall shear stresses are known. The assessment for the momentum-exchange method for three-dimensional flows will be made first in this case. Finally, the momentum-exchange method will be evaluated by considering the drag on a sphere due to a uniform flow over a sphere in a finite domain. The details for the flow field computation can be found in Refs. $[28,11]$.

3.1. Two-dimensional pressure-driven channel flow. In the case of the channel flow, the force on the top wall $(y=H)$ at a given location $x\left(i=N_{x} / 2+1\right.$, for example) can be evaluated using the 


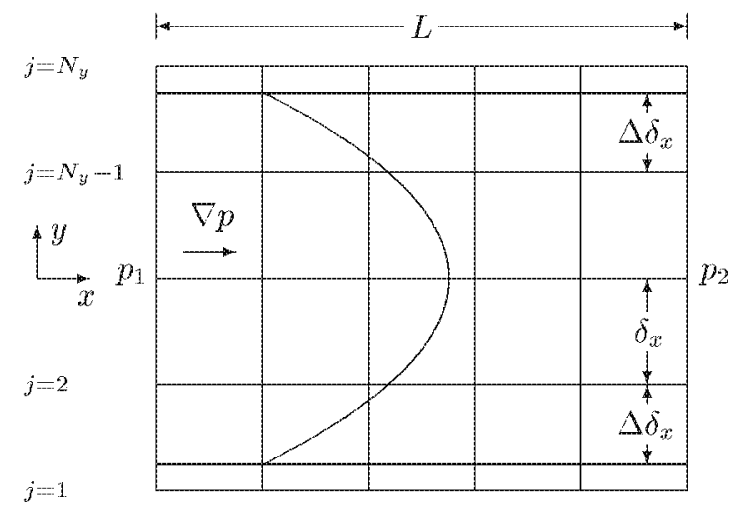

FIG. 4. The channel flow configunation in the LBE simulations with an arbitrary $\triangle$.

momentum-exchange method as follows. The wall is located between $j=N_{y}$ and $N_{y}-1$ (cf. Fig. 4 ). The $x$ and $y$ components of the force on the fluid at the top wall near the ith node are

$$
\begin{aligned}
F_{x}= & {\left[\tilde{f}_{6}(i, j)+\tilde{f}_{2}(i-1, j-1)\right] e_{6, x}+\left[\tilde{f}_{8}(i, j)+\tilde{f}_{4}(i+1, j-1)\right] e_{8, x} } \\
F_{y}= & {\left[\tilde{f}_{6}(i, j)+\tilde{f}_{2}(i-1, j-1)\right] e_{6, y}+\left[\tilde{f}_{8}(i, j)+\tilde{f}_{4}(i+1, j-1)\right] e_{8, y} } \\
& +\left[\tilde{f}_{7}(i, j)+\tilde{f}_{3}(i, j-1)\right] e_{7, y}
\end{aligned}
$$

where $e_{\alpha, j}$ denotes the $j$ th Cartesian component of velocity $e_{\alpha}$. Since $\delta_{x}=1, F_{x}$ and $F_{y}$ are, effectively, the total shear and normal stresses, $\sigma_{x y}$ and $\sigma_{y y}$, which include the pressure and the deviatoric stresses, on the fluid element at $y=H$.

Based on Eq. (2.7), the deviatoric component of the fluid shear stresses at $j=N_{y}-1$ (or $\left.y=N_{y}-3+\Delta\right)$ and $N_{y}-2$ (or $y=N_{y}-4+\Delta$ ) can be exactly evaluated based on the nonequilibrium part of the distribution functions in the flow field if they are correctly given. A linear extrapolation of the deviatoric shear stresses to $y=H=N_{y}-3+2 \Delta$ yields

$$
\tau_{x y, w)}^{(\mathrm{neq})}=\tau_{x y}\left(j=N_{y}-1\right)+\Delta\left[\tau_{x y}\left(j=N_{y}-1\right)-\tau_{x y}\left(j=N_{y}-2\right)\right]
$$

where the superscript "(neq)" denotes the value computed from $f_{\alpha}^{\text {(neq) }}$, and the subscript $w$ refers to the value at the wall. The deviatoric normal stress, $\tau_{y y, w}^{(\text {neq })}$, can be similarly computed. In a fully developed channel flow, the normal component of the deviatoric stress $\tau_{y y}(y)$ is expected to be zero while the total normal stress $\sigma_{y y}(y)$ is equal to the negative of the pressure $(-p)$. It needs to be pointed out that this method of evaluating $\tau_{x y, w}^{(\text {neq })}$ given by Eq. (3.2) for two-dimensional channel flow is equivalent to the method of the surface stress integration based on the extrapolated pressure and the deviatoric stresses on the solid wall except that no numerical integration on the solid surface is needed.

After the velocity profile $u_{x}(y)$ is obtained from $f_{\alpha}$, the shear stress $\tau_{z y}$ on the wall can also be calculated using the near wall velocity profile as

$$
\begin{aligned}
\left.\rho \nu \frac{d u_{x}}{d y}\right|_{y=H}= & \rho \nu \frac{(2+\Delta)}{(1+\Delta)} \frac{\left[0-u_{x}\left(j=N_{y}-1\right)\right]}{\Delta} \\
& -\rho \nu \frac{\Delta}{(1+\Delta)}\left[u_{x}\left(j=N_{y}-1\right)-u_{x}\left(j=N_{y}-2\right)\right] .
\end{aligned}
$$

In the above, a linear extrapolation is employed to evaluate the velocity derivative $\left.\left(d u_{x} / d y\right)\right|_{y=I I}$ at the wall. 
TABLE 3.1

Comparison of fuid stresses at $y=M$ in a two-dimenstonal pressure driven channel flow with dp/dx $=-1.0 \times 10^{-6}$ in

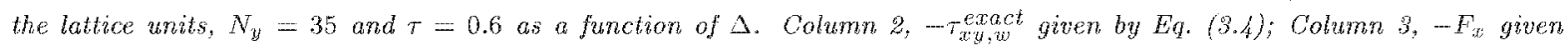
by Eq. (3.1a); Column 4, $-\tau_{x y, w}^{(\text {neq }}$ given by Eq. (3.2); Column 5, - pu(dux/dy)|y=H Eq. (3.3): Column 6, -Fy given by Eq. (3.1b); Column 7, pressure p obtained in the simulation.

\begin{tabular}{|lcccccc|}
\hline$\Delta$ & $-\tau_{x y, w}^{\text {exact }} \times 10^{5}$ & $-F_{x} \times 10^{5}$ & $-\tau_{x y, w}^{\text {neq }} \times 10^{5}$ & $-\left.\rho \nu \frac{d u_{x}}{d y}\right|_{y=l l} \times 10^{5}$ & $-F_{y}$ & $p$ \\
\hline 0.01 & 1.601 & 1.6333 & 1.6010 & 3.5294 & 0.3333 & 0.3333 \\
\hline 0.02 & 1.602 & 1.6333 & 1.6020 & 2.5555 & 0.3333 & 0.3333 \\
\hline 0.03 & 1.603 & 1.6333 & 1.6030 & 2.2309 & 0.3333 & 0.3333 \\
\hline 0.04 & 1.604 & 1.6333 & 1.6040 & 2.0685 & 0.3333 & 0.3333 \\
\hline 0.05 & 1.605 & 1.6333 & 1.6050 & 1.9710 & 0.3333 & 0.3333 \\
\hline 0.1 & 1.610 & 1.6333 & 1.6100 & 1.7760 & 0.3333 & 0.3333 \\
\hline 0.2 & 1.620 & 1.6333 & 1.6200 & 1.6781 & 0.3333 & 0.3333 \\
\hline 0.25 & 1.625 & 1.6333 & 1.6250 & 1.6583 & 0.3333 & 0.3333 \\
\hline 0.3 & 1.630 & 1.6333 & 1.6300 & 1.6451 & 0.3333 & 0.3333 \\
\hline 0.3333 & 1.633 & 1.6333 & 1.6330 & 1.6385 & 0.3333 & 0.3333 \\
\hline 0.35 & 1.635 & 1.6333 & 1.6350 & 1.6357 & 0.3333 & 0.3333 \\
\hline 0.4 & 1.640 & 1.6333 & 1.6400 & 1.6285 & 0.3333 & 0.3333 \\
\hline 0.5 & 1.650 & 1.6333 & 1.6500 & 1.6184 & 0.3333 & 0.3333 \\
\hline 0.6 & 1.660 & 1.6333 & 1.6600 & 1.6214 & 0.3333 & 0.3333 \\
\hline 0.7 & 1.670 & 1.6333 & 1.6700 & 1.6244 & 0.3333 & 0.3333 \\
\hline 0.8 & 1.680 & 1.6333 & 1.6800 & 1.6274 & 0.3333 & 0.3333 \\
\hline 0.9 & 1.690 & 1.6333 & 1.6900 & 1.6305 & 0.3333 & 0.3333 \\
\hline 0.95 & 1.695 & 1.6333 & 1.6950 & 1.6321 & 0.3333 & 0.3333 \\
\hline 0.99 & 1.699 & 1.6333 & 1.6990 & 1.6335 & 0.3333 & 0.3333 \\
\hline \hline
\end{tabular}

Finally, the exact solution for the fluid shear stress on the wall $(y=H)$ is

$$
\tau_{x y, w}^{\mathrm{exact}}=\frac{1}{2} \frac{d p}{d x} H, \quad H=N_{y}-3+2 \Delta
$$

based on the parabolic velocity profile or simple control volume analysis. This exact result can be used to assess the accuracy of the aforementioned methods for the force evaluation.

In the LBE simulations, the pressure gradient is enforced through the addition of an equivalent body force after the collision step $[26,11]$. While the velocity field given by the LBE solution can be unique, the pressure field [thus the density field $\rho(x, y)$ ] can only be unique up to an arbitrary constant. In view of Eq. (3.3), it is difficult to compare the stresses for different cases if $\rho(i, j)$ converges to different values in each case. To circumvent this difficulty, the density field in the channel flow simulation is normalized by $\rho\left(i=2, j=N_{y} / 2\right)$ at every time step. This normalization procedure results in $\rho(x, y)=1$ throughout the entire computational domain. It is also applied to the three-dimensional flow in a circular pipe.

Table 3.1 compares the numerical values of the shear stress for a typical case $\left(N_{y}=35, d p / d x=-10^{-6}\right.$ in the lattice units, and $\tau=0.6)$ based on: $\tau_{x y, w}^{\text {exact }}$ given by Eq. (3.4), $F_{x}$ given by Eq. (3.1a), $\tau_{x y, w}^{\text {(neq) }}$ given by Eq. (3.2), and $\left.\rho \nu\left(d u_{x} / d y\right)\right|_{y=I I}$ given by Eq. (3.3). Also listed is the comparison between $F_{y}$ given by Eq. (3.1b) and $-p$. All computations are carried out with double precision accuracy.

It is noted that $\tau_{x y, w}^{(\text {neq) }}$ is identical to $\tau_{x y, w}^{\text {exact }}$ for all values of $\Delta$. A closer examination of the shear 
stress profile using Eq. (2.7) across the channel reveals that $\tau_{x y, u}^{(\text {req }}(y)$ is also equal to the exact shear stress profile $\tau_{x y}^{\text {exact }}(y)$, which is linear, despite the errors in the velocity profile $u_{x}(y)$ for all values of $\Delta$. A linear extrapolation, Eq. (3.2), for a linear profile therefore gives the exact wall shear stress. Thus, the exactness of $\tau_{x y, w}^{(\mathrm{neg})}$ in the LBE simulation of chamel flow indicates the reliability of the LBE solution for the stress field $\tau_{i j}^{\text {(neq) }}(x, y)$ by using Eq. (2.7). However, as Fig. 3 indicates, the accuracy of integrating $\tau_{i j}^{\text {(neq) }}(x, y)$ to obtain the fluid dynamic force in nontrivial geometries needs to be further investigated, as will be discussed in the following sections.

For $0<\Delta<1$, the normal force $F_{y}$ given by Eq. (3.1b) based on the momentum-exchange method agrees exactly with the pressure on the wall. This is a rather special quantity since deviatoric component of the force is identically zero. Nevertheless, the method of the momentum exchange does give a reliable value for the normal stress.

For the shear (tangential) force, it is observed from Table 3.1 that for fixed $d p / d x, F_{x}$ does not change as $\Delta$ increases from 0.01 to 0.99 . On the other hand, the exact result $\tau_{x y, w}^{\text {exact }}=\frac{1}{2}(d p / d x)\left(N_{y}-3+2 \Delta\right)$, increases linearly with $\Delta$. Further computations were carried out over a range of $N_{y}(=35,67,99$, and $131)$ and $\tau(=0.505,0.51,0.52,0.6,0.7,0.8,0.9,1.0,1.2,1.4$, and 1.6). The results indicate that the momentum-exchange method gives the shear stress on the top wall as

$$
F_{x}=\frac{1}{2} \frac{d p}{d x}\left(N_{y}-3+\frac{2}{3}\right)
$$

That is, $F_{x}$ is independent of $\tau$ and $\Delta$. The error in $F_{x}$ is zero when $\Delta=1 / 3$. The absolute error attains the maximum when $\Delta=1$, which gives the relative error of $4 / 3 H$ for $F$. Although the frequently used momentum-exchange method is a natural choice for the force evaluation in conjunction with the bounce-back boundary condition for $\Delta=1 / 2$, one must be aware that this method is not exact and the error in the force evaluation using the momentum-exchange method depends on $\Delta$ and the resolution.

The error in $F_{x}$ is due to the fact that the derivatives of the velocity field are not considered in the boundary conditions. This can be understood by analyzing Eq. (3.1a). At the steady state, and with the approximation that

$$
\tilde{f}_{\alpha} \approx f_{\alpha}^{(\mathrm{eq})}+f_{\alpha}^{(1)}=f_{\alpha}^{(\mathrm{eq})}-\frac{1}{\tau} w_{\alpha} \frac{3}{c^{2}}\left(\boldsymbol{e}_{\alpha} \cdot \nabla\right)\left(e_{\alpha} \cdot u\right)
$$

Equation (3.1a) at the top wall becomes

$$
F_{x} \approx 2 w_{2} \rho \frac{3}{c^{2}} e_{2} \cdot\left(u_{f f}+u_{f}-2 u_{w}\right)
$$

where the substitution of Eq. (2.4) for $\tilde{f}_{6}$ and $\tilde{f}_{8}$ has been made. The only term in the above equation which has $\Delta$ dependence is $u_{b f}$. When $0 \leq \Delta 1 / 2, F_{x}$ is independent of $\Delta$, and when $1 / 2 \leq \Delta<1, F_{x}$ weakly depends on $\Delta$ because $u_{w}=0$ in this case [see Eqs. (2.3)]. In the case where $F_{x}$ is obtained by summing over a set of symmetric lattice points, cancellations in the summation may further weaken the dependence of $F_{x}$ on $\Delta$.

Table 3.1 also shows that for the shear stress based on the derivative of the velocity obtained by using finite-difference, the loss of accuracy is quite significant for small values of $\Delta(\leq 0.05)$ when $\tau=0.6$. For other values of $\Delta(\geq 0.3)$, the accuracy is comparable with that of $F_{x}$. However, as shown in Fig. $5(a)$, the accuracy of $\left.\rho \nu\left(d u_{x} / d y\right)\right|_{y=\Pi}$ based on the near-wall velocity derivative deteriorates as the relaxation time $\tau$ increases (from 0.51 to 1.6 ). To see the cause of the increasing error in $\left.\rho \nu\left(d u_{x} / d y\right)\right|_{y=I}$, Fig. $5(b)$ shows dimensionless wall velocity, $u_{w} / u_{c}$, obtained by a three-point second-order Lagrangian extrapolation 

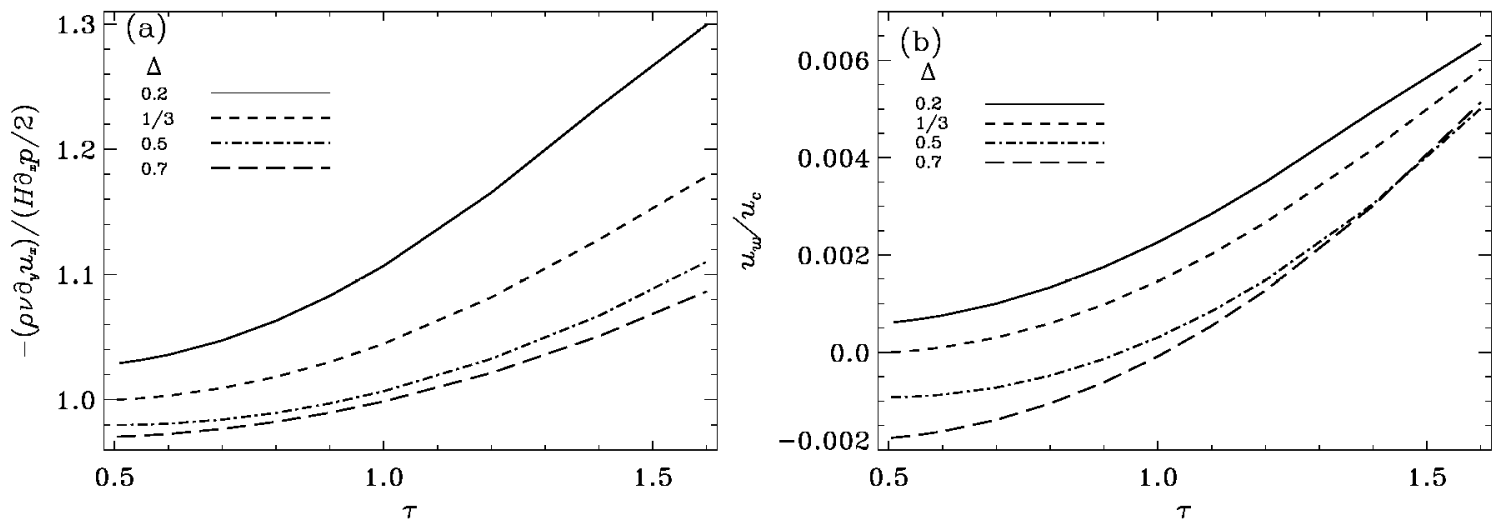

FIG. 5. The LBE simulations of the channel flow, with $\Delta=0.2,1 / 3,0.5$, and 0.7 . The pressure drop is $\partial_{k} p=-1.0 \times 10^{-6}$ in latice units. (a) Ratio between the wall force, $\left.\rho \nu \partial_{y} u_{x}\right|_{y=H}$, evaluated by using Eq. (3.3), and the exact value $-\tau_{z y}^{\text {exact }}=$ $-H \partial_{x} p / 2$, given by Eq. (3.4) as a function of $\tau$. (b) Normalized wall slip velocity $u_{w} / u_{t}$ as a function of $\tau$.

of the near wall velocity $u_{x}(y)$ as a function of $\tau$. The increasing slip velocity $u_{w}$ on the wall with the increasing relaxation time $\tau$ was also observed in Ref. [15]. It is the result of increasing particle mean free path that causes the deviation of the kinetic solution from the hydrodynamic solution. It is clear that the poor performance of $\left.\rho \nu\left(d u_{x} / d y\right)\right|_{y=I I}$ is associated with the increasing error in the near wall velocity profile as $\tau$ increases. Since the stress tensor $\tau_{i j}$ can be calculated directly from $f_{\alpha}$ [see Eq. (2.7)] without the need for directly computing velocity derivatives, the force evaluation method based on the evaluation of the velocity gradient in the form of Eq. (2.6) is not recommended.

3.2. Steady uniform flow over a column of cylinders. For a uniform flow over a column of circular cylinders of radius $r$ and center-to-center distance $H$ (see the left part of Fig. 6 for illustration), symmetry conditions for $f_{\alpha}$ 's are imposed at $y= \pm H / 2$. Most of the details of flow field simulation can be found in Ref. [28]. The Reynolds number is defined by the diameter of the cylinder $d$ as $\operatorname{Re}=U d / \nu$, where $U$ is the uniform velocity in the inlet. It must be noted that for a consistent determination of the force, the upstream boundary must be placed far upstream. A shorter distance between the cylinder and the boundary will result in higher drag. In this study, it is placed at about 20 radii to the left of the center of the cylinder. Reducing the distance between the boundary and the cylinder to 12.5 radii while keeping the rest of the computational parameters fixed would increase the drag coefficient by about $1.8 \%$ at Re $=100$. The downstream boundary is located about $25-30$ radii behind the cylinder to allow sufficient wake development. The simulation is terminated when the following criterion based on the relative $L_{2}$-norm error in the fluid region $\Omega$ is satisfied,

$$
E_{2}=\sqrt{\frac{\sum_{x_{i} \in \Omega}\left\|u\left(x_{i}, t+1\right)-u\left(x_{i}, t\right)\right\|^{2}}{\sum_{x_{i} \in \Omega}\left\|u\left(x_{i}, t+1\right)\right\|^{2}}} \leq \epsilon .
$$

In this case, $\epsilon=10^{-6}$ was chosen for both $\operatorname{Re}=10$ and 100 .

Following Fornberg [38], the drag coefficient over a circular cylinder of radius $r$ is defined as

$$
C_{D}=\frac{\left|F_{x}\right|}{\rho U^{2} r}
$$

Figure 7(a) compares $C_{D}$ obtained from: momentum-exchange method, surface stress integration, and finite difference result of Fornberg [38] using a vorticity-stream function formulation at $\operatorname{Re}=100, \mathrm{H} / \mathrm{r}=20$, and 

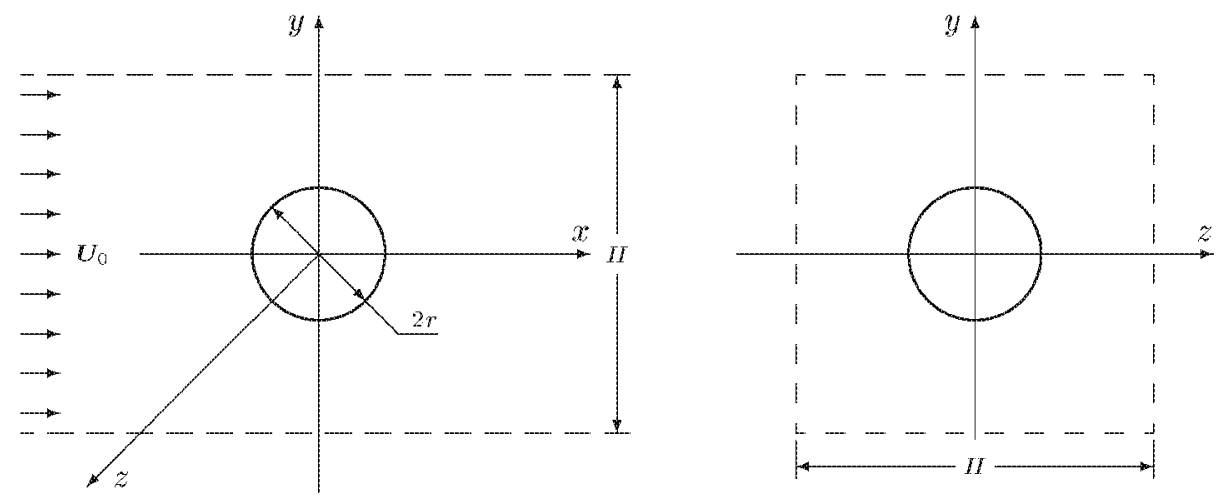

FIG. 6. Computational domain for the uniform flow past of sphere of radius $r$. The dashed lines indicate boundaries of computational domain. (left) Unbounded domain in xy plone, and (right) bounded domain in yz plane.

radius $r$ ranging from 2.8 to 13.2 . For $r>8$, both the momentum exchange and the stress integration methods give satisfactory results for $C_{D}$ in comparison with the value of 1.248 given in Ref. [38]. The small differences in $C_{D}$ could be due to the fact that in Ref. [38], the computational domain is much larger in the downstream direction - the downstream boundary condition is imposed at 300 radii behind the cylinder in Ref. [38], as opposed to 25 - 30 radii here. This adds credence to the validity of Eq. (2.9) for evaluating the total force on a body. The values of $C_{D}$ from the momentum-exchange method have a little less variation than that from the stress integration. Accepting an error of less than $5 \%$, reliable data for $C_{D}$ can be obtained, using the momentum-exchange method, for $r>5$. That is, ten lattice spacings across the diameter of the cylinder are necessary to obtain reliable values of the force. This is consistent with the finding by Ladd [14]. In the range of $5<r<7$, the stress-integration method produces larger fuctuations in the results than the momentum exchange method. For smaller radius, i.e., coarser resolutions, while both methods give poor results (due to insufficient resolution), the stress integration method yields much larger errors.

Figure $7(\mathrm{~b})$ compares $C_{D}$ obtained from the methods of momentum exchange and the stress integration for $R e=10$. The momentum-exchange method seems to gives a converged result at larger $r(>8)$. Based on the data for $r>8$, an average value of $C_{D} \approx 3.356$ is obtained. In contrast, the stress-integration method has a larger fluctuation than the large $r$ result from the momentum-exchange method even for $r>8$. Averaging over the results for $r>8$, the stress integration gives $C_{D} \approx 3.319$. The difference between converged results of two methods is about $1 \%$. For $r$ less than or around 5 , the fluctuation in $C_{D}$ from the stress-integration method is much larger than that in the momentum-exchange method. The conclusions from the comparisons in Fig. 7 are as follows: (i) both methods for force evaluation can give accurate results; (ii) the momentumexchange method gives more consistent drag; and (iii) in the range of $10<R e<100$, a resolution of ten lattice spacings across the diameter of the cylinder are needed in order to obtain consistent and reliable drag values. In other words, the lattice (grid) Reynolds number $\operatorname{Re}^{*}(=U / \nu)$ should be less than 10 in the calculations.

In the above results presented in Figs. $7(a)$ and $7(b)$, the center of the cylinder is placed on a lattice grid, thus the computational mesh is symmetric with respect to the geometry of the cylinder. To test the effect of the mesh symmetry on the accuracy of the force evaluation, the calculation of the flow at $R e=10$ is repeated with different values of the cylinder center offset $\Lambda_{x}$ in the $x$ direction, or $\Lambda_{y}$ in the $y$ direction. The radius of the cylinder is deliberately chosen to be only 6.4 lattice grids. In order to preserve the mirror 

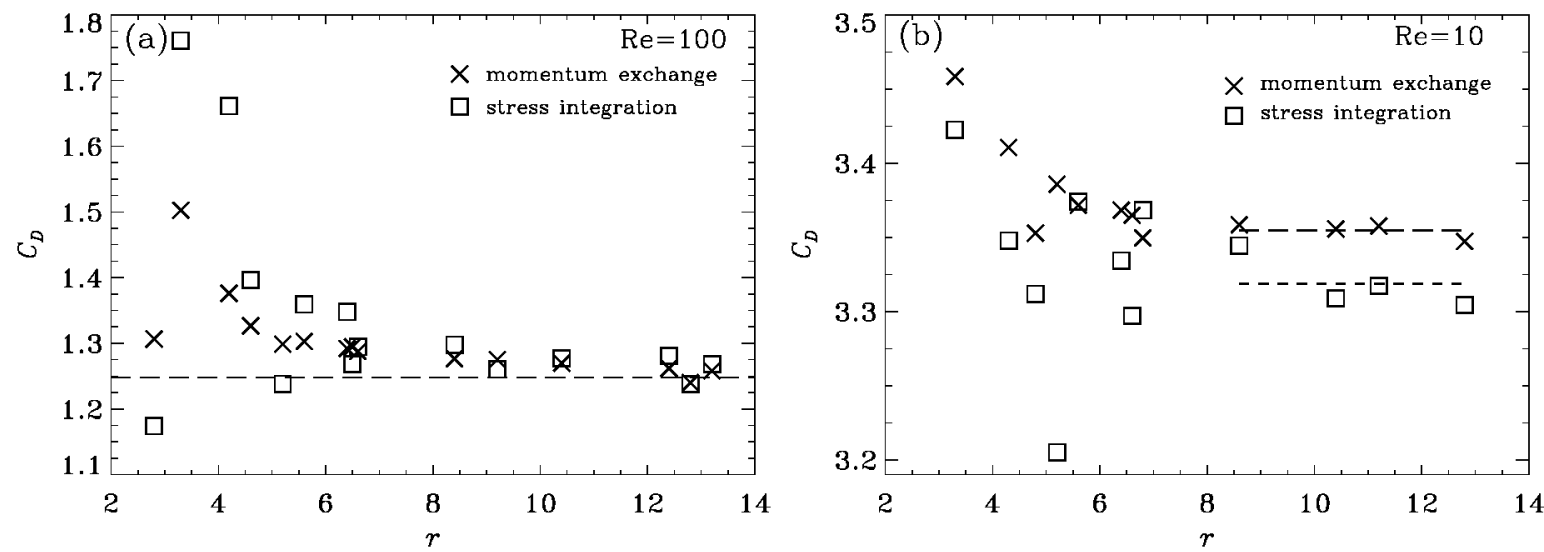

FIG. 7. The drag coefficient for a uniform flow past a column of cylknders ower a range of radius $r$. (a) Pe $=100$. The dashed line indicates the value of $C_{D}=1.24$ obtained in Ref. [387. (b) Re $=10$. The dashed lines indicate the values of $C_{D}$ averaged over 4 largest radii.

symmetry of the flow in the $y$ direction, we use different boundary conditions for upper and lower boundaries (at $y= \pm H / 2$ ). For $\Lambda_{y}=0$ while varying $\Lambda_{x}$, we use the symmetric boundary conditions, which maintain the flow symmetry with respect to the center line in the $x$ direction. For $\Lambda_{x}=0$ while varying $\Lambda_{y}$, we use the periodic boundary conditions at $y= \pm H / 2$, which are equivalent to the symmetric boundary conditions when $\Lambda_{y}=0$, but better reflect the flow symmetry when $\Lambda_{y} \neq 0$. The results of the drag coefficient $C_{D}$ are presented in Table 3.2. The variation of $C_{D}$ due to the change of the center of cylinder offset from a grid point is less than $1 \%$ when the cylinder diameter is only about 13 lattice spacings. The outcome is consistent with the expected truncation errors caused by mesh perturbation. We notice that the variation in $C_{D}$ due to $\Lambda_{x}$ is about one order of magnitude smaller than that due to $\Lambda_{y}$. This is precisely because when $\Lambda_{y}=0$ the mesh symmetry coincides with the flow symmetry in the $y$ direction, and when $\Lambda_{y} \neq 0$ the mesh symmetry is lost. This asymmetry due to $\Lambda_{y} \neq 0$ results in the change of the lift coefficient from $O\left(10^{-14}\right)$ to $O\left(10^{-2}\right)$, which is the same order of magnitude of the variation in $C_{D}$. It is our observation that the accuracy of the force evaluation schemes used here is dictated by that of the boundary conditions at the solid walls. The error due to symmetry of the computational mesh with respect to the geometry of an object is well bounded. This is also observed in other independent studies [23,33].

TABLE 32

The effect of symmetry of the computational mesh on the force evaluation for the steady uniform flow over a column of cylinders. The Reynolds number $\operatorname{Re}=10(\tau=0.6)$, the radius of the cylinder $r=6.4$ (in the lattice unit of $\left.\delta_{x}=1\right)$, and $H / r=20$. The variation of $C_{D}$ due to the change of the center of cylinder offset from a grid point is less than $1 \%$.

\begin{tabular}{|cccccc|}
\hline \multicolumn{5}{|c|}{$\Lambda_{x}=0$, periodic boundary conditions at $y= \pm H / 2$} \\
\hline$\Lambda_{y}$ & 0 & 0.2 & 0.4 & 0.6 & 0.8 \\
\hline$C_{D}$ & 3.3661 & 3.3637 & 3.3526 & 3.3526 & 3.3637 \\
\hline \hline \multicolumn{4}{|c}{$\Lambda_{y}=0$, symmetric boundary conditions in $y= \pm H / 2$} \\
\hline$\Lambda_{x}$ & 0 & 0.2 & 0.4 & 0.6 & 0.8 \\
\hline$C_{D}$ & 3.3661 & 3.3666 & 3.3646 & 3.3667 & 3.3692 \\
\hline
\end{tabular}

It is worth noting that the wall shear stress in the channel flow obtained by using the method of 
momentum exchange has a relative error proportional to the resolution acouss the charnel. For a resolution of 10 - 20 lattice spacings across the diameter considered here, the relative error in the drag appears, however, smaller than in the channel flow case. At Re $=100$, with $r>10$, the average value of the drag obtained by using the method of momentum exchange has a 1.7\% relative exror comparing with Fornberg's data [38]. If the boundary layer thickness is estimated roughly to be $3 \times 2 \% / \sqrt{\text { Re }} \approx 6$, there are only about six lattice spacings across the boundary layer over which the velocity profile changes substantially. Based on the insight from the channel flow result, it is possible that the deviatoric shear stresses on the surface of the cylinder that are effectively incorporated in the method of momentum exchange suffer comparable levels of error as in the channel flow. The effective error cancellation over the entire surface of the body may have contributed to the good convergence behavior in the drag shown in Figs. $7($ a) and $7(b)$.

3.3. Flow over an asymmetrically placed circular cylinder in channel with vortex shedding. Schäfer and Turek [39] reported a set of benchmark results for a laminar flow over a circular cylinder of radius $r$ that is asymmetrically placed inside a channel. In the present study, $r=12.8$ is used and the center of the cylinder coincides with a grid point. The distance from the center of the cylinder to the upper wall and lower wall is $h_{+}=4.2 r$ and $h_{-}=4.0 r$, respectively. This results in $\Delta_{+}=0.76$ for the upper wall and $\Delta_{-}=0.2$ for the lower wall, respectively. The channel inlet has a parabolic profile and is placed at four radii upstream of the cylinder center according to the specification of the benchmark test [39], This results in $\Delta=0.2$ for the inlet boundary. A zeroth-order extrapolation for $f_{a}$ is used at the exit boundary that is located 40 radii downstream of the cylinder center. Thus there are a total of $564 \times 105$ square lattices in the How field. For $\mathrm{Re}=2 r \bar{U} / \nu=100$ based on the average inlet velocity $\bar{U}$, the use of relaxation time $\tau=0.55$ requires $U \approx 0.0651$.

At this Reynolds number, the flow becomes unsteady and periodic vortex shedding is observed. Figures $8(\mathrm{a}), 8(\mathrm{~b})$, and $8(\mathrm{c})$, respectively, show time-dependent behaviors of the lift coefficient

$$
C_{L}=\frac{F_{y}}{\rho U^{2} p}
$$

and the drag coefficient $C_{D}[$ see $\mathrm{Eq} .(3.9)]$, and the pressure difference

$$
\Delta P=\frac{p_{f}-p_{t}}{\rho_{0} U^{2}}
$$

where $p_{f}$ and $p_{b}$ are the pressures at the front and the back of the cylinder, respectively, and $\rho_{0}$ is the constant density imposed at the entrance. The data of $C_{L}, C_{D}$, and $\triangle P$ are compared with the benchmark results in Ref. [39]. We first note that the present numerical value of Strouhal number $\mathrm{St}=2 \mathrm{r} / \bar{U} T$ is about 0.3033 , where $T$ is the period of the lift curve. This agrees very well with the range of St values $(0.2950-0.3050)$ given in Ref. [39]. We note that the difference in $C_{L}(t)$ between the momentum-exchange method and the surface stress-integration method is indiscernible graphically. For the drag coefficient $C_{D}(t)$, it is interesting to note that although there is about $0.25 \%$ difference between the results given by the momentum-exchange method and the surface stress-integration method, both methods of force evaluation give two peaks in the $C_{D}(t)$ curves. Physically, these two peaks in the $C_{D}(t)$ curve correspond to the existence of a weaker vortex and a stronger vortex alternately shed behind the cylinder. The difference in the strength of the vortices results from the difference: $h_{+} / r=4.2$ and $h-. / r=4.0$ in the passages between the cylinder and the channel walls. There is no report on the occurrence of these two peaks in Ref. [39]. Instead, a range of the maximum $C_{D}$ (from 3.22 to 3.24 ) by different researchers was given. The present value of the higher peak is well within the range. It is interesting to note that both peaks of $C_{D}(t)$ obtained by the momentum-exchange method 

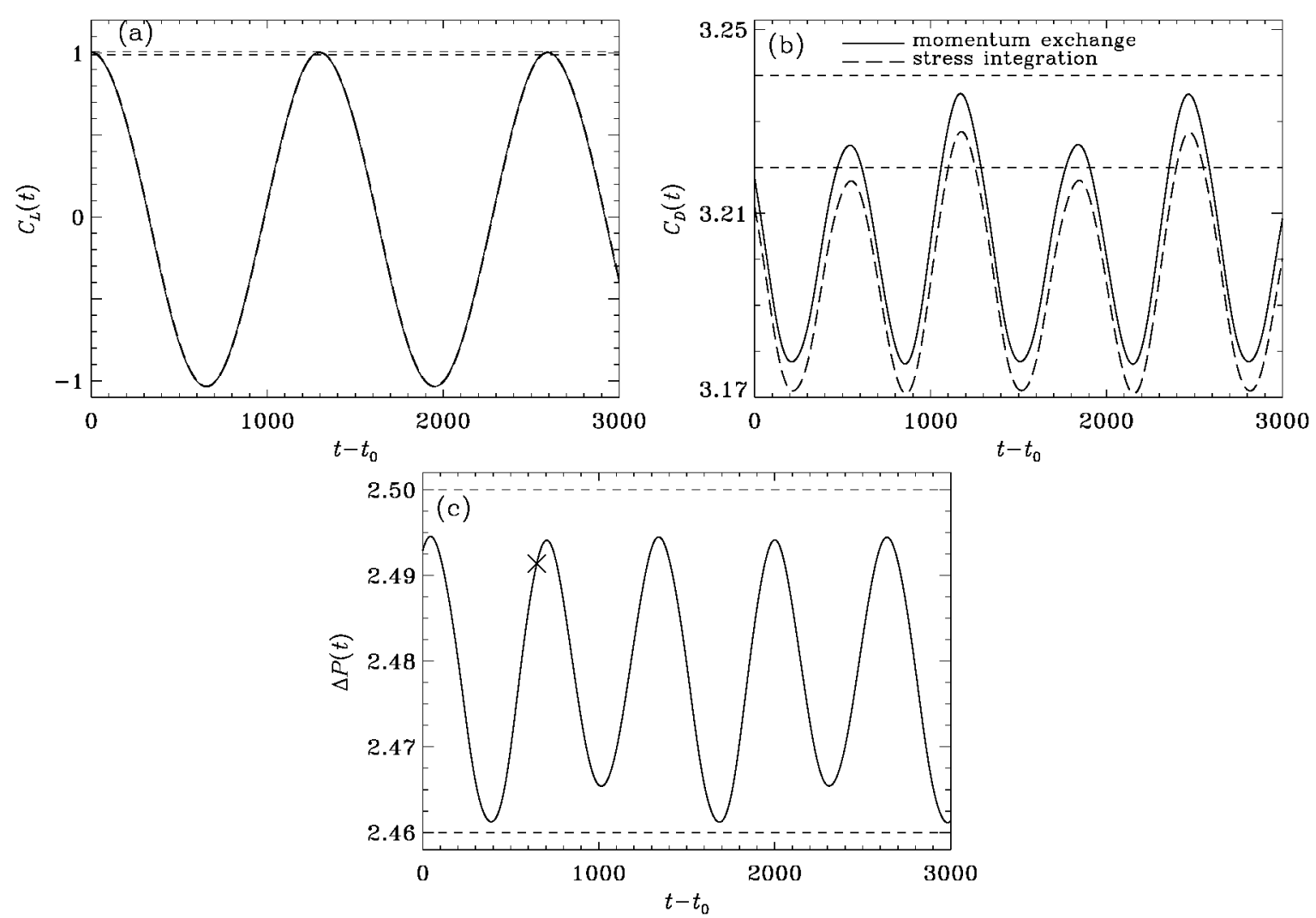

FIG. 8. The $2 D$ flou past a cylinder asymmetrically placed in a chennel. The variations of the lift coefficient $C_{L}$, the drag coefficient $C_{D}$, and the pressure difference $\triangle P$ as functions of time $t$ (after an iritial run time to) are compared with the benchmark results in Ref. $139 \%$. At the time to, the lift coefficient $C_{L}(t)$ attains its maximum value $C_{L}^{\text {max. }}$. The dashed horizontal lines indicate the upper and lower bounds in Ref. [39]. The solid and dashed curves are the results obtained by using momentum exchange and stress integration, respectively. (a) The lift coefficient $C_{L}(t)$. Note that the results obtained by using the two methods are indistinguishable on the graph. (b) The drag coefficient $C_{D}(t)$. (c) The pressure difference $\triangle P(t)$. The symbol $x$ indicates the value of $\Delta P\left(t_{0}+T / 2\right)$ given in Table 3.8 , where $T(\approx 1296.5)$ is the period of $C_{L}(t)$.

are also within the range, as shown in Fig. $8(\mathrm{~b})$. A further refined computation of the present problem using a multiblock procedure [40] with $r=40$ in the fine grid region yield nearly the same results for $C_{D}(t)$ and $C_{L}(t)$

We compile in Table 3.3 the values of Strouhal number St, maximum and minimum drag coefficient $C_{D}^{\max }$ and $C_{D}^{\min }$, maximum and minimum lift coefficient $C_{L}^{\max }$ and $C_{L}^{\min }$, and the pressure difference $\Delta P$ obtained by the LBE methods and other schemes of computational fluid dynamics given in Ref. [39]. The value of $\Delta P$ is measured at $t_{0}+T / 2$, where $t_{0}$ is the moment when $C_{L}(t)$ reaches its maximum value $C_{L}^{\max }$, and $T$ is the periodicity of $C_{L}(t)$. For the LBE simulations, $T$ is between 1296 and 1297 (in the lattice unit of $\delta_{t}=1$ ). We use $T=1296.5$ in the determination of the Strouhal number St. With a resolution much coarser than those used in Ref. [39], the LBE results are well within the bounds given in Ref. [39]. This clearly demonstrates the accuracy of the lattice Boltzmann method.

3.4. Pressure-driven flow in a circular pipe. The steady-state flow field was obtained by using D3Q19 model with $\tau=0.52$ [11]. Eq. (2.9) is used to evaluate the force on the boundary points along the circumference of the pipe over a distance of one lattice in the axial direction. The resulting axial force $F_{x}$ 
TABLE 3.3

Values of $S t, C_{D}^{\max }, C_{D}^{\text {min }}, C_{L}^{\text {max }}, C_{L}^{\text {min }}$, and $\Delta P$ for the flow over a sD cylinder asymmetrically placed in a channel. "Momentum" and "Stress" denote, respectively, the momentum-exchange method and the stress-integration method in the LBE calculations. The CFD results are the bounds in Ref. $\left[397\right.$, which does not have dato for $C_{D}^{\mathrm{min}}$ and $C_{L}^{\mathrm{min}}$.

\begin{tabular}{|lcccccc|}
\hline \hline \multicolumn{1}{|c}{ method } & $\mathrm{St}$ & $C_{D}^{\max }$ & $C_{D}^{\text {main }}$ & $C_{L}^{\text {max }}$ & $C_{L}^{\min }$ & $\Delta P$ \\
\hline Momentum & 0.3033 & 3.2358 & 3.1771 & 1.0045 & -1.0347 & 2.4914 \\
\hline Stress & 0.3033 & 3.2275 & 3.1708 & 1.0040 & -1.0340 & 2.4914 \\
\hline CFD & $0.2950-0.3050$ & $3.2200-3.2400$ & - & $0.9900-1.0100$ & - & $2.4600-2.5000$ \\
\hline
\end{tabular}

is, equivalently, the force given by $\tau_{w} 2 \pi r \delta_{x}$, where $\tau_{w}$ is the wall shear stress and $r$ is the pipe radius. For a fully developed flow inside a circular pipe, the exact fluid shear stress at the pipe wall is given by

$$
\tau_{w}^{\text {exact }}(2 \pi r)=\pi r^{2} \frac{d p}{d x}
$$

We examine the normalized axial force,

$$
\eta=\frac{F_{x x}}{\pi r^{2} \frac{d p}{d x}}
$$

Figure 9 shows the normalized coefficient $\eta$ over a range of $r: 3.5-23.5$. Except for $r \leq 5, \eta$ is rather close to 1. It was noticed in Ref. [11] that the accuracy of LBE solution for the pipe flow is not as good as that for the two-dimensional channel flow due to the distribution of values of $\Delta$ around the pipe. The accuracy of the drag is dictated by the accuracy of the flow field if the force evaluation method is exact. For the pipe flow, the error in $F_{x}$ results from the inaccuracy in the flow field and the errors in the force evaluation scheme based on momentum exchange (as seen in the previous section for the two-dimensional channel flow case). For $r>5$, the largest error in $F_{x}$ is about $3.5 \%$ and it occurs at $r=15.5$. Again, there is no systematic error in $F_{x}$. Given the complexity of the boundary in this three-dimensional flow, the results shown in Fig. 9 are satisfactory in the sense that it adds further credence to the momentum-exchange method for force evaluation.

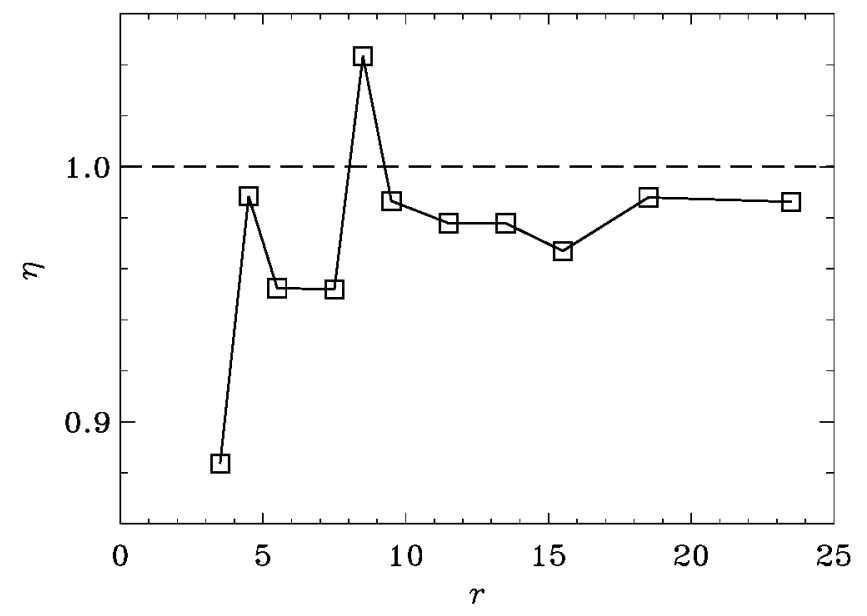

Fig. 9. The ratio $\eta$ between the tangential force $F x$ on the pipe and its exact walue ( $r r^{2} \nabla p$ ) over a range of pipe radius $r$. 
3.5. Steady uniform How over a sphere. To limit the computational effort, a finite domain of $-H / 2 \leq y \leq H / 2$ and $-H / 2 \leq z \leq H / 2$, with $H / \gamma^{*}=10$ is used to compute the flow past a sphere of radius $r$ (see Fig. 6). Two cases are considered: (a) the flow past a single sphere, and (b) the flow over a two-dimensional array of spheres (all located at $x=0$ ) with the center of the spheres forming square lattices. In the former case, the boundary conditions at $j_{y}=1\left(y=H / 2\right.$ corresponds to $\left.j_{y}=2\right)$ for $f_{\alpha}$ 's are given by the following linear extrapolation

$$
f_{\alpha}\left(j_{x}, 1, j_{z}\right)=2 f_{\alpha}\left(j_{x}, 2, j_{z}\right)-f_{\alpha}\left(j_{x}, 3, j_{z}\right)
$$

The velocity at $j_{y}=2$ is set as

$$
u\left(j_{x}, 2, j_{z}\right)=u\left(j_{x}, 3, j_{z}\right)
$$

Similar treatment is applied at $y=H / 2$ and $z= \pm H / 2$. In the latter case, symmetry conditions are posed on $f_{\alpha}$ 's at $j_{y}=1$ by using the values of $f_{\alpha}$ 's at $j_{y}=3$ (see Ref. [28] for the two-dimensional case). At the inlet, a uniform velocity profile is imposed at $j_{x}=1.5$ (half way between the first and second lattices). The upstream boundary is located at 7.5 radii to the left of the sphere center in all simulations.

For flow over a sphere, the drag coefficient is often expressed as

$$
C_{D}=\frac{F_{x}}{\frac{1}{2} \rho U^{2} \pi r^{2}}=\frac{24}{\operatorname{Re}} \phi, \quad \phi=-\frac{F_{x}}{6 \pi r U \rho \nu},
$$

where $\phi$ accounts for the non-Stokesian effect of the drag. For two types of the boundary conditions at $(y= \pm H / 2$ and $z= \pm H / 2), \phi_{s}$ denotes the non-Stokesian correction for the case where the symmetry conditions are imposed at $(y= \pm H / 2$ and $z= \pm H / 2)$ and $\phi_{\infty}$ denotes the results for the case where the extrapolation for $f_{\alpha}$ is used at $(y= \pm H / 2$ and $z= \pm H / 2)$ in order to simulate the unbounded flow.

Figure 10(a) shows the non-Stokesian coefficient $\phi_{\infty}$ for $r=3.0,3.2,3.4,3.6,3.8,4.0,5.1,5.2,5.4,5.6$, and 5.8 , for $H / r=10$ at $R e=10$. The relaxation time is $r=0.7$. With this range of $r$, the number of the boundary nodes on the surface of the sphere increases roughly by a factor of $(5.8 / 3)^{2} \approx 3.74$; the actual counts of the boundary nodes $x_{b}$ gives a ratio $2370 / 546=4.35$. The largest difference is $1.9 \%$ between $r=3.0$ and $r=3.2$ that have the least resolution in the cases investigated. For a uniform flow over an unbounded sphere, an independent computation using a finite difference method based on the vorticity-stream function formulation with high resolution gives a drag coefficient $\phi \approx 1.7986$ at Re $=10$. The largest difference between this result and the LBE results is $1.36 \%$ at $r=3.2$. If the LBE data for the drag is averaged over the range of $r$, one obtains $\phi \approx 1.8086$, which differs from 1.7986 by $0.54 \%$. Hence, the LBE solutions with $3.0 \leq r \leq 5.8$ yield very consistent values for the drag force. Figure $10(b)$ shows the non-Stokesian correction factor $\phi_{s}$ for a uniform flow over a planar array of spheres for $3.0 \leq r \leq 5.8$ and $H / r=10$, at $\operatorname{Re}=10$. It is important to note that with the improvement of the surface resolution by a factor of 4.35 , there is little systematic variation in $\phi_{s}(r)$. The largest deviation from the average value, $\bar{\phi}_{s} \approx 1.963$, is $1.1 \%$ at $r=5.0$. It is clear that the LBE solution gives reliable fluid dynamic forces on a sphere at $r \approx 3.5$ for a moderate value of Re. The set of data for $\phi_{s}$ is inherently more consistent than that for $\phi_{\infty}$ since the symmetry boundary condition can be exactly specified at $y= \pm H / 2$ and $z= \pm H / 2$, while the extrapolation conditions given by Eqs. (3.12) and (3.13) do not guarantee the free stream condition at $y= \pm H / 2$ and $z= \pm H / 2$. Yet, both $\phi_{\infty}$ and $\phi_{s}$ exhibit remarkable self-consistency from coarse to not-so-coarse resolutions.

4. Conclusions. Two methods for evaluating the fluid force in conjunction with the method of lattice Boltzmann equation for solving fluid flows involving curved geometry have been examined. The momentumexchange method is very simple to implement. It is shown in the channel flow simulation that momentumexchange method is not an exact method. The error in the wall shear stress is inversely proportional to 

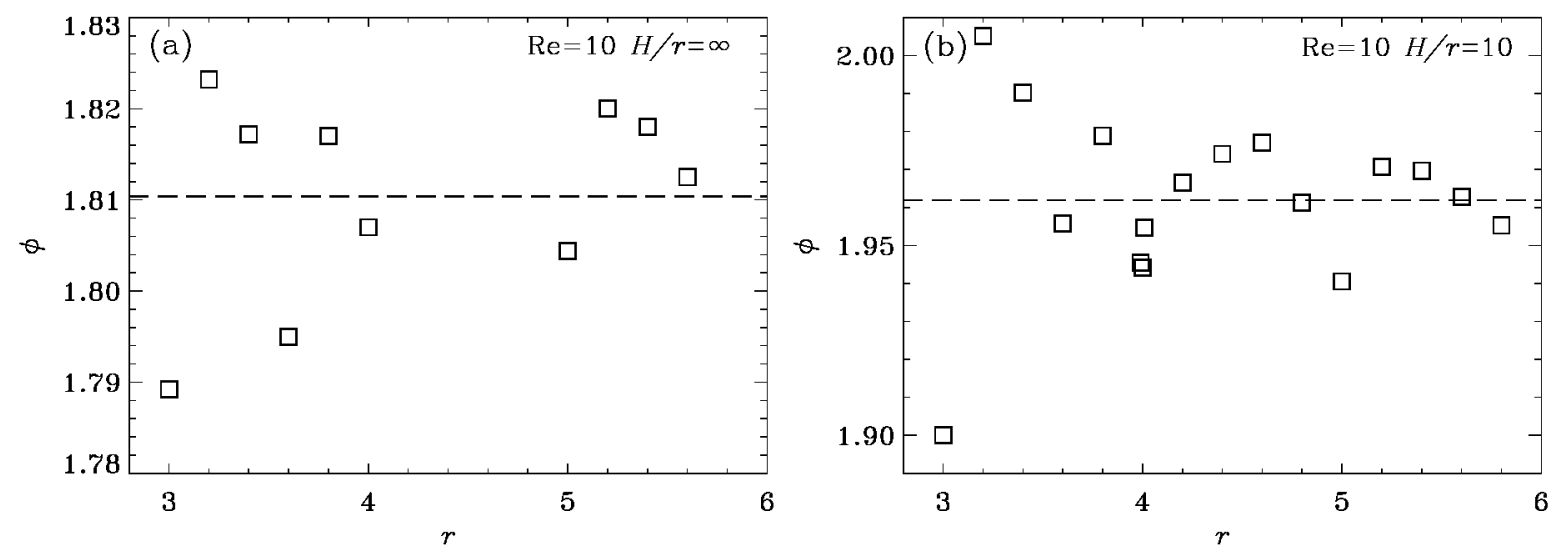

Fig. 10. Flow past sphere. Variation of the non-Stokesian correction factor $\phi=-F \cdot / 6 \pi r U p \nu$ as a function of sphere radius $r$ at $R e=10$. The dashed lines are values of $\phi(r)$ averaged over $r$. (a) The flow past a single sphere in an unbounded field $(H / r=\infty)$. (b) The flow past a planar array of spheres $(H / r=10)$.

the resolution. In two- and three-dimensional flows over a bluff body, it can give accurate drag values when there are at least ten lattice spacings across the body at $R e \sim 100$. The method of integrating the stresses on the surface of the body gives similar results when there is sufficient resolution but it exhibits much larger fluctuations than that in the method of momentum exchange when the resolution is limited. In addition, the stress-integration method requires considerably more efforts in implementing the extrapolation and integration on the body surface in comparison with the method of momentum exchange.

It is interesting to note that the momentum-exchange method is perhaps superior to the stress-integration method because the former method is directly based on the distribution functions while the latter is derived from further processing of the distribution functions. In addition, the momentum-exchange method uses interpolations while the stress-integration method uses extrapolations. Often extrapolations are more noisy and unstable than interpolations. Even with a coarse resolution that does not yield very accurate local information, accurate force evaluation can be accomplished with the lattice Boltzmann method. Among the two force evaluation methods, the method of momentum exchange is recommended for force evaluation on curved boundaries for its simplicity, accuracy, and robustness.

Acknowledgments. The authors are grateful to Prof. A.J.C. Ladd for his helpful comments. This work is partially supported by NASA Langley Research Center under the program of Innovative Algorithms for Aerospace Engineering Analysis and Optimization. R. Mei also acknowledges partial support of the Engineering Research Center (ERC) for Particle Science and Technology at the University of Florida, the National Science Foundation (EEC-9402989), and Industrial partners of the ERC.

\section{Appendix A. LBE Models in Two and Three Dimensions.}

The nine-velocity (or 9-bit) LBE model on a two-dimensional square lattice, denoted as the D2Q9 model, has been widely used for simulations of two-dimensional flows. For three-dimensional flows, there are several cubic lattice models, such as the fifteen-velocity (D3Q15), nineteen-velocity (D3Q19), and twenty-sevenvelocity (D3Q27) models, which have been used in the literature [10]. All these models have a rest particle (with zero velocity) in the discretized velocity set $\left\{e_{\alpha} \mid a=0,1, \ldots,(b-1)\right\}$. For athermal fluids, the equilibrium distributions for the D2Q9, D3Q15, D3Q19, and D3Q27 models are all of the following form 
$[6,7]$

$$
f_{\alpha}^{(\mathrm{eq})}=w_{\alpha} \rho\left[1+\frac{3}{c^{2}}\left(e_{\alpha} \cdot u\right)+\frac{9}{2 c^{4}}\left(e_{\alpha} \cdot u\right)^{2}-\frac{3}{2 c^{2}} u^{2}\right],
$$

where $w_{\alpha}$ is a weighting factor and $e_{\alpha}$ is a discrete velocity, $c \equiv \delta_{x} / \delta_{t}$ is the unit speed, and $\delta_{x}$ and $\delta_{t}$ are the lattice constant and the time step, respectively. The discrete velocities for the D2Q9 models are

$$
e_{\alpha}= \begin{cases}(0,0), & \alpha=0 \\ ( \pm 1,0) c,(0, \pm 1) c, & \alpha=1,3,5,7 \\ ( \pm 1, \pm 1) c, & \alpha=2,4,6,8\end{cases}
$$

and the values of the weighting factor $w_{\alpha}$ are

$$
w_{\alpha}= \begin{cases}\frac{4}{9}, & \alpha=0 \\ \frac{1}{9}, & \alpha=1,3,5,7 \\ \frac{1}{36}, & \alpha=2,4,6,8\end{cases}
$$

For the D3Q19 model, the discrete velocities are

$$
e_{\alpha}= \begin{cases}(0,0), & \alpha=0 \\ ( \pm 1,0,0) c,(0, \pm 1,0) c,(0,0, \pm 1) c, & \alpha=1-6 \\ ( \pm 1, \pm 1,0) c,(0, \pm 1, \pm 1) c,( \pm 1,0, \pm 1) c, & \alpha=7-18\end{cases}
$$

and the weighting factor $w_{\alpha}$ is given by $[7]$

$$
w_{\alpha}= \begin{cases}\frac{1}{3}, & \alpha=0, \\ \frac{1}{18}, & \alpha=1-6 \\ \frac{1}{36}, & \alpha=7-18 .\end{cases}
$$

The discrete velocity sets $\left\{\boldsymbol{e}_{\alpha}\right\}$ for the D2Q9 and D3Q19 models are shown in Fig. 1.

The density and velocity can be computed from $f_{\alpha}$,

$$
\begin{aligned}
\rho & =\sum_{\alpha} f_{\alpha}=\sum_{\alpha} f_{\alpha}^{\left(\mathrm{eq}_{\mathrm{q}}\right)} \\
\rho u & =\sum_{\alpha} e_{\alpha} f_{\alpha}=\sum_{\alpha} e_{\alpha} f_{\alpha}^{(\mathrm{eq})} .
\end{aligned}
$$

The speed of sound of the above LBE models is

$$
c_{s}=\frac{1}{\sqrt{3}} c
$$

and the equation of state is that of an ideal gas such that

$$
p=c_{s}^{2} \rho
$$

The viscosity of the fluid is

$$
\nu=c_{s}^{2} \lambda
$$

for the discrete velocity model of Eq. (1.2). It should be noted that the equilibrium distribution function $f_{\alpha}^{(e q)}$ is in fact a Taylor series expansion of the Maxwellian $f^{(0)}[6,7]$. This approximation of $f_{\alpha}^{(\mathrm{eq})}$ in algebraic form makes the LBE method valid only in the incompressible flow limit $u / c \rightarrow 0$. 
Equation (1.2) is often discretized in space $x$ and time $t$ into the lattice Boltzmann equation

$$
f_{\alpha}\left(x_{i}+e_{\alpha} \delta_{t}, t+\delta_{t}\right)-f_{\alpha}\left(x_{i}, t\right)=-\frac{1}{\tau}\left[f_{\alpha}\left(x_{i}, t\right)-f_{\alpha}^{(\mathrm{eq})}\left(\boldsymbol{x}_{i}, t\right)\right],
$$

where $\tau=\lambda / \delta_{t}$. For this LBGK model [1,2], the viscosity in the Navier-Stokes equation derived from the above lattice Boltzmann equation is

$$
\nu=\left(\tau-\frac{1}{2}\right) c_{s}^{2} \delta_{t}
$$

The $-1 / 2$ correction in the above formula for $\nu$ comes from the second-order derivatives of $f_{\alpha}$ when $f_{\alpha}\left(x_{i}+\right.$ $e_{\alpha} \delta_{t}, t+\delta_{t}$ ) in Eq. (A.8) is expanded in a Taylor series in $u$. This correction in $v$ makes the lattice Boltzmann method formally a second-order method for solving incompressible flows [7]. Obviously, the physical and computational stabilities require that $\tau>1 / 2$.

\section{REFERENCES}

[1] H. Chen, S. Chen, And W.H. Matthaeus, Recovery of the Navier-Stokes equations using a latticegas Boltzmann method, Phys. Rev. A, 45 (1992), pp. R5339-R5342.

[2] Y.H. QIAN, D. D'HUMières, AND P. LALlEMAND, Lattice BGK models for Navier-Stokes equation, Europhys. Lett., 17 (1992), pp. 479-484.

[3] R. BEnzI, S. SUCCI, AND M. Vergassola, The lattice Boltzmann equation: Theory and applications, Phys. Rep., 222 (1992), pp. 145-197.

[4] S. Chen And G.D. Doolev, Lattice Boltzmann method for fluid flows, Annu. Rev. Fluid Mech., 30 (1998), pp. 329-364.

[5] P.L. Bhatnagar, E.P. Gross, AND M. Krook, A model for collision processes in gases. I. Small amplitude processes in charged and neutral one-component system, Phys. Rev., 94 (1954), pp. $511-$ 525.

[6] X. HE AND L.-S. Luo, A priori derivation of the lattice Boltzmann equation, Phys. Rev. E, 55 (1997), pp. R6333-R6336.

[7] X. HE AvD L.-S. Luo, Theory of the lattice Boitzmann method: From the Boltzmann equation to the lattice Boltzmann equation, Phys. Rev. E, 56 (1997), pp. 68116817.

[8] T. ABE, Derivation of the lattice Boltzmann method by means of the discrete ordinate method for the Boltzmann equation, J. Comput. Phys., 131 (1997), pp. 241-246.

[9] X. SHAN AND X. HE, Discretization of the velocity space in the solution of the Boltzmann equation, Phys. Rev. Lett., 80 (1998), pp. 65-68.

[10] Y.H. QIAN, S. SUCCI, AND S.A. ORsZAG, Recent advances in lattice Boltzmann computing, in Annual Reviews of Computational Physics, Vol. III, edited by D. Stauffer, (World Scientific, Singapore, 1995), pp. 195-242.

[11] R. MEI, W. ShyY, D. YU, AND L.-S. LUo, Lattice Boltzmann method for 3-d flows with curved boundary, J. Comput. Phys., 161 (2000), pp. 680-699.

[12] D.P. ZreGLER, Boundary conditions for lattice Boltzmann simulations, J. Stat. Phys, 71 (1993), pp. $1171-1177$.

[13] I. Ginziourg And P.M. Alder, Boundary flow condition analysis for the three-dimensional laltice Boltzmann model, J. Phys. II, 4 (1994), pp. 191214. 
[14] A.J.C. LADD, Numerical simulation of particular suspensions wia a discretized Boltzmann equation, Part 2, Numerical results, J. Fluid Mech., 271 (1994), pp. 311-339.

[15] D.R. Noble, S. Cmen, J.G. Geokgradis, And R.O. Buctuvs, A consistent hydrodynamic boundary condition for the lattice Boltzmann method, Phys. Fluid, 7 (1995), pp. 203-209.

[16] O. BEHREND, Solid boundaries in particle suspension simulations via lattice Boltzmann method, Phys. Rev. E, 52 (1995), pp. 1164-1175.

[17] T. Inamuro, M. Yoshino, AND F. OGINo, A non-slip boundary condition for lattice Boltzmann simulations, Phys. Fluids, 7 (1995), pp. 2928-3000.

[18] S. Chen, D. MARTíNez, AND R. MEI, On boundary conditions in lattice Boltzmann method, Phys. Fluids, 8 (1996), pp. 2527-2536.

[19] I. GINzBURG AND D. D'Humièes, Local second-order boundary methods for lattice Boltzmann models, J. Stat. Phys., 84 (1996), pp. 927-971.

[20] X. HE, Q. ZOU, L.-S. LUO, AND M. DEMBO, Analytic solutions and analysis on non-slip boundary condition for the lattice Boltzmann BGK model, J. Stat. Phys., 87 (1997), pp. 115-136.

[21] L.-S. LUO, Analytic solutions of linearized lattice Boltzmann equation for simple flows, J. Stat. Phys., 88 (1997), pp. 913926.

[22] Q. ZOU AND X. HE, On pressure and velocity boundary conditions for the lattice Boltzmann BGK model, Phys. Fluids, 9 (1997), pp. 1591-1598.

[23] M. Bouzidi, M. Firdaouss, And P. Lallemand, Momentum transfer of a Boltzmann-lattice fluid with boundaries, Phys. Fluids, 13 (2001), pp. 452-3459.

[24] A.J.C. LADD AND R. VERBERG, Lattice Boltzmann simulations of particle-fluid suspensions, J. Stat. Phys., 104 (2001), pp. 1191-1251.

[25] R. MEI AND W. SHYY, On the finite difference-based lattice Boltzmann method in curvilinear coordinates, J. Comput. Phys., 143 (1998), pp. 426-448.

[26] X. HE AND G. DOOLEN, Lattice Boltzmann method on curvilinear coordinates systems: Flow arownd a circular cylinder, J. Comput. Phys., 134 (1997), pp. $306-315$.

[27] O. FILIPPova AND D. HÄNEL, Grid refinement for lathice-BGK models, J. Comput. Phys., 147 (1998), pp. $219-228$.

[28] R. MeI, L.-S. LuO, AND W. SHYY, An accurate curved boundary treatment in the lattice Boltzmann method, J. Comput. Phys., 155 (1999), pp. $307-330$.

[29] M.N. Kogan, Rarefied Gas Dynamics (Plenum, New York, 1969).

[30] S. HARRIS, An Introduction to the Theory of the Boltzmann Equation (Holt, Rinehart and Winston, New York, 1971).

[31] C. Ceroignan, The Boltzmann Equation and its Applications (Springer-Verlag, New York, 1988).

[32] R. Cornubert, D. D'Humieres, And D. Levefiore, A Knudsen layer theory for lattice gases, Physica D, 47 (1991), pp. 241-259.

[33] D. D'Humitres, M. BouzD, AND P. LALlemand, Thinten-velocity 30 lattice Boltzmann model, Phys. Rev. E, 63 (2001), 066702.

[34] O. Fmiprova (private communication).

[35] W. SHYY, Computational Modeling for Flud Flow and Interfacial Thansport, revised printing (Elsevier, Amsterdam, 1994).

[36] W. ShyY, S.S. ThakUR, H. OUYANG, J. LiU, AND E. Blosch, Computational Techniques for Complex Transport Phenomena (Cambridge University Press, Cambridge, 1997). 
[37] S. Thakur, W. SHYY, H.S. Udaykumar, AND L. Hil, Multiblock interface treatments in a pressurebased flow solver, Numer. Heat Transfer, Part B, 33 (1998), pp. 367-396.

[38] B. Fonnberg, Steady incompressible flow past a row of circular cylinder, J. Fluid Mech., 225 (1991), pp. $655-671$.

[39] M. SCHÄFER AND S. TUREK, Benchmark computations of laminar flow over a cylinder, in Flow Simulation with High-Performance Computers 11 , edited by E.H. Hirschel, Notes in Numerical Fluid Mechanics (Vieweg, Braunschweig, 1996), Vol. 52, pp. 547-566.

[40] D. YU, R. MEI, AND W. SHYY, A multi-block lattice Boltzmann method for fluid flows, to appear in Int. J. Numer. Methods Fluids (2002). 


\begin{tabular}{|c|c|c|c|}
\hline \multicolumn{3}{|c|}{ REPORT DOCUMENTATION PAGE } & $\begin{array}{l}\text { Form Approved } \\
\text { OMB No. 0704-0188 }\end{array}$ \\
\hline \multicolumn{4}{|c|}{ 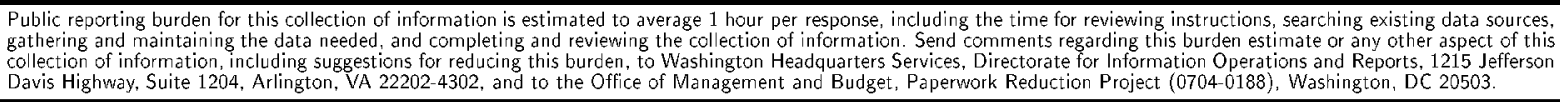 } \\
\hline 1. AGENCY USE ONLY(Leave blank) & $\begin{array}{l}\text { 2. REPORT DATE } \\
\text { July } 2002\end{array}$ & \multicolumn{2}{|c|}{$\begin{array}{l}\text { 3. REPORT TYPE AND DATES COVERED } \\
\text { Contractor Report }\end{array}$} \\
\hline \multicolumn{3}{|c|}{$\begin{array}{l}\text { 4. TITLE AND SUBTITLE } \\
\text { FORCE EVALUATION IN THE LATTICE BOLTZMANN METHOD } \\
\text { INVOLVING CURVED GEOMETRY }\end{array}$} & \multirow[t]{2}{*}{$\begin{array}{l}\text { 5. FUNDING NUMBERS } \\
\begin{array}{l}\text { C NAS1-97046 } \\
\text { WU } 505-90-52-01\end{array}\end{array}$} \\
\hline \multicolumn{3}{|c|}{$\begin{array}{l}\text { 6. AUTHOR(S) } \\
\text { Renwei Mei, Dazhi Yu, Wei Shyy, and Li-Shi Luo }\end{array}$} & \\
\hline \multicolumn{2}{|c|}{$\begin{array}{l}\text { ICASE } \\
\text { Mail Stop 132C } \\
\text { NASA Langley Research Center } \\
\text { Hampton, VA 23681-2199 }\end{array}$} & & $\begin{array}{l}\text { REPORT NUMBER } \\
\text { ICASE Report No. } 2002-22\end{array}$ \\
\hline \multicolumn{2}{|c|}{$\begin{array}{l}\text { 9. SPONSORING/MONITORING AGENCY NAME(S) AND ADDRESS(E } \\
\text { National Aeronautics and Space Administration } \\
\text { Langley Research Center } \\
\text { Hampton, VA 23681-2199 }\end{array}$} & $\begin{array}{l}\text { 10. SPONS } \\
\text { AGENC } \\
\text { NASA } \\
\text { ICASF }\end{array}$ & $\begin{array}{l}\text { 10. SPONSORING/MONITORING } \\
\text { AGENCY REPORT NUMBER } \\
\text { NASA/CR-2002-211662 } \\
\text { ICASE Report No. } 2002-22\end{array}$ \\
\hline \multicolumn{4}{|c|}{$\begin{array}{l}\text { 11. SUPPLEMENTARY NOTES } \\
\text { Langley Technical Monitor: Dennis M. Bushnell } \\
\text { Final Report } \\
\text { To appear in Physical Review E. }\end{array}$} \\
\hline \multicolumn{2}{|c|}{$\begin{array}{l}\text { 12a. DISTRIBUTION/AVAILABILITY STATEMENT } \\
\text { Unclassified-Unlimited } \\
\text { Subject Category } 34 \\
\text { Distribution: Nonstandard } \\
\text { Availability: NASA-CASI (301) 621-0390 }\end{array}$} & & 12b. DISTRIBUTION CODE \\
\hline \multicolumn{4}{|c|}{$\begin{array}{l}\text { 13. ABSTRACT (Maximum } 200 \text { words) } \\
\text { The present work investigates two approaches for force evaluation in the lattice Boltzmann equation: the momentum- } \\
\text { exchange method and the stress-integration method on the surface of a body. The boundary condition for the particle } \\
\text { distribution functions on curved geometries is handled with second order accuracy based on our recent works. The } \\
\text { stress-integration method is computationally laborious for two-dimensional flows and in general difficult to implement } \\
\text { for three-dimensional flows, while the momentum-exchange method is reliable, accurate, and easy to implement for } \\
\text { both two-dimensional and three-dimensional flows. Several test cases are selected to evaluate the present methods, } \\
\text { including: (i) two-dimensional pressure-driven channel flow; (ii) two-dimensional uniform flow past a column of } \\
\text { cylinders; (iii) two-dimensional flow past a cylinder asymmetrically placed in a channel (with vortex shedding); } \\
\text { (iv) three-dimensional pressure-driven flow in a circular pipe; and (v) three-dimensional flow past a sphere. The } \\
\text { drag evaluated by using the momentum-exchange method agrees well with the exact or other published results. }\end{array}$} \\
\hline \multirow{2}{*}{\multicolumn{3}{|c|}{$\begin{array}{l}\text { 14. SUBJECT TERMS } \\
\text { lattice Boltzmann method, force evaluation on fluid-solid interface, momentum-exchange } \\
\text { method, stress-integration method, boundary condition for curved geometries, accuracy, } \\
\text { 3-D flows }\end{array}$}} & \begin{tabular}{|c} 
15. NUMBER OF PAGES \\
28
\end{tabular} \\
\hline & & & $\begin{array}{r}\text { 16. PRICE CODE } \\
\mathrm{A} 03\end{array}$ \\
\hline $\begin{array}{l}\text { 17. SECURITY CLASSIFICATION } \\
\text { OF REPORT } \\
\text { Unclassified }\end{array}$ & $\begin{array}{l}\text { 18. SECURITY CLASSIFICATION } \\
\text { OF THIS PAGE } \\
\text { Unclassified }\end{array}$ & $\begin{array}{l}\text { 19. SECURITY CLASSIFICATION } \\
\text { OF ABSTRACT }\end{array}$ & $\begin{array}{l}\text { 20. LIMITATION } \\
\text { OF ABSTRACT }\end{array}$ \\
\hline
\end{tabular}

